Supporting Information

\title{
A GAP Replacement: Improved Synthesis of 3-Azidooxetane and Its Homopolymer Based on Sulfonic Acid Esters of Oxetan-3-ol
}

\author{
Max Born, Konstantin Karaghiosoff and Thomas M. Klapötke* \\ Department of Chemistry and Biochemistry, Energetic Materials Research, Ludwig- \\ Maximilian University, Butenandtstr. 5-13 (D), 81377 Munich, Germany.
}

*E-Mail: tmk@cup.uni-muenchen.de 


\section{Table of contents}

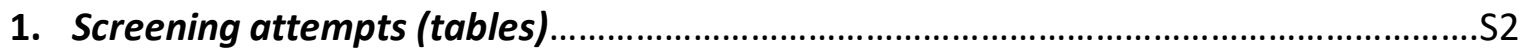

2. NMR Spectra of prepared compounds.........................................................................

3. X-ray Diffraction and Hirshfeld analysis.....................................................................

4. Heat of formation calculation and thermal analysis.................................................S14

5. Detonation parameters and hot-plate test.............................................................

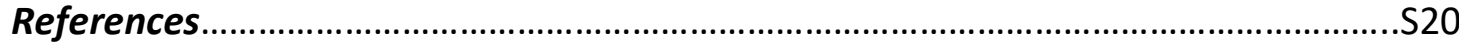

\section{Screening attempts}

Table S1. Reaction condition screening for 3-tosyloxyoxetane (1).

\begin{tabular}{|c|c|c|c|c|c|c|}
\hline Batch & $\begin{array}{l}\text { Oxetan-3-ol (g, } \\
\text { mmol) }\end{array}$ & $\begin{array}{l}\text { Base } \\
\text { (mmol, eq.) }\end{array}$ & $\begin{array}{l}p \text {-TsCl } \\
\text { (mmol, eq.) }\end{array}$ & $\begin{array}{l}t(\mathrm{~h}), \mathrm{T} \\
\left({ }^{\circ} \mathrm{C}\right)\end{array}$ & $\begin{array}{l}\text { Crude } \\
\text { yield (\%) }\end{array}$ & $\begin{array}{l}\text { Solvent, } \\
\text { volume }[\mathrm{mL}]\end{array}$ \\
\hline A & $2.00,27.0$ & $\mathrm{NaOH}(29.7,1.1)$ & $28.4,1.05$ & 1,50 & 13 & Water, 5.30 \\
\hline B & $1.00,13.5$ & $\mathrm{NaOH}(14.9,1.1)$ & $14.2,1.05$ & 2,50 & 70 & Water, 2.65 \\
\hline C & $0.50,6.75$ & Pyridine $(8.10,1.2)$ & $7.42,1.1$ & 5 , reflux & 0 & $\mathrm{CHCl}_{3}, 20.0$ \\
\hline D & $0.25,3.37$ & $\mathrm{~K}_{2} \mathrm{CO}_{3}(6.75,2.0)$ & $3.71,1.1$ & 5 , reflux & 36 & DCM, 50.0 \\
\hline $\mathbf{E}$ & $2.00,27.0$ & $\operatorname{TEA}(29.7,1.1)$ & $27.0,1.0$ & 4 , reflux & 53 & $\mathrm{CHCl}_{3}, 50.0$ \\
\hline $\mathbf{F}$ & $2.00,27.0$ & $\operatorname{TEA}(29.7,1.1)$ & $27.0,1.0$ & 6 , reflux & 42 & $\mathrm{CHCl}_{3}, 50.0$ \\
\hline G & $1.00,13.5$ & $\operatorname{TEA}(14.9,1.1)$ & $13.5,1.0$ & $24, \mathrm{rt}$. & $68 / 57^{*}$ & DCM, 25.0 \\
\hline H & $2.00,27.0$ & $\operatorname{TEA}(29.7,1.1)$ & $29.7,1.1$ & 24, reflux & 32 & DCM, 50.0 \\
\hline I & $4.00,54.0$ & $\operatorname{TEA}(59.4,1.1)$ & $59.4,1.1$ & 4 , reflux & $83 / 75^{*}$ & DCM, 100 \\
\hline
\end{tabular}

* Yield of pure material after recrystallization.

Table S2. Base screening for the preparation of 3-mesyloxyoxetane (2).

\begin{tabular}{lllllll}
\hline Batch & $\begin{array}{l}\text { Oxetan-3-ol (g, } \\
\mathbf{m m o l})\end{array}$ & $\begin{array}{l}\text { Base } \\
\text { (mmol, eq.) }\end{array}$ & $\begin{array}{l}\mathbf{M s C l} \\
(\mathbf{m m o l}, \text { eq. })\end{array}$ & $\begin{array}{l}\mathbf{t}(\mathbf{h}), \\
\mathbf{T}\left({ }^{\circ} \mathbf{C}\right)\end{array}$ & $\begin{array}{l}\text { Crude } \\
\text { yield (\%) }\end{array}$ & $\begin{array}{l}\text { Solvent, } \\
\text { volume [mL }]\end{array}$ \\
\hline I & $0.50,6.75$ & $\operatorname{DBU}(13.5,2.0)$ & $7.42,1.1$ & $3, \mathrm{rt}$. & 30 & $\mathrm{DCM}, 15.0$ \\
II & $0.50,6.75$ & $\operatorname{DABCO}(6.75,2.0)$ & $7.42,1.1$ & $3, \mathrm{rt}$. & 87 & $\mathrm{DCM}, 15.0$ \\
III & $0.30,4.05$ & $\operatorname{NaHMDS}(8.19,2.0)$ & $4.46,1.1$ & $3, \mathrm{rt}$. & 23 & $\mathrm{MeCN}, 15.0$ \\
\hline
\end{tabular}

The azidation step was roughly screened using 3-tosyloxyoxetane (1) as model compound because it was found to be less prone to substitution than 3-mesyloxyoxetane (2). The reactions were monitored by TLC and ${ }^{1} \mathrm{H}$ NMR spectroscopy was used to qualitatively determine whether the turnover was low $(<30 \%)$, medium (30-60\%), high ( $>60 \%$ ) or even quantitative. Overall, DMSO gave the best result and was the most environmentally friendly compared to other solvents. 
Table S3. Use of different azidation agents and solvents to prepare 3-azidooxetane.

\begin{tabular}{llll}
\hline Solvent & Azidation agent & $\mathbf{t}(\mathbf{h}), \mathbf{T}\left({ }^{\circ} \mathrm{C}\right)$ & Conversion \\
\hline Chloroform & $\mathrm{TBAA}^{*}$ & 24, reflux & very low \\
THF & $\mathrm{TBAA}$ & 24, reflux & very low \\
MeCN & $\mathrm{NaN}_{3}$, 18-crown-6 & 96 , reflux & low \\
1,4-Dioxane/water 9:1 & $\mathrm{NaN}_{3}$ & 24, reflux & low \\
Acetone/water 1:1 & $\mathrm{NaN}_{3}$ & 36, reflux $\left(80^{\circ} \mathrm{C}\right)$ & medium \\
Sulfolane & $\mathrm{NaN}_{3}$ & $36,100{ }^{\circ} \mathrm{C}$ & high \\
DMF & $\mathrm{NaN}_{3}$ & $36,100^{\circ} \mathrm{C}$ & quantitative \\
DMSO & $\mathrm{NaN}_{3}$ & $36,100^{\circ} \mathrm{C}$ & quantitative \\
\hline
\end{tabular}

* TBAA = tetrabutylammonium azide

\section{NMR Spectra}

\section{Oxetan-3-yl 4-methylbenzenesulfonate (compound 1)}

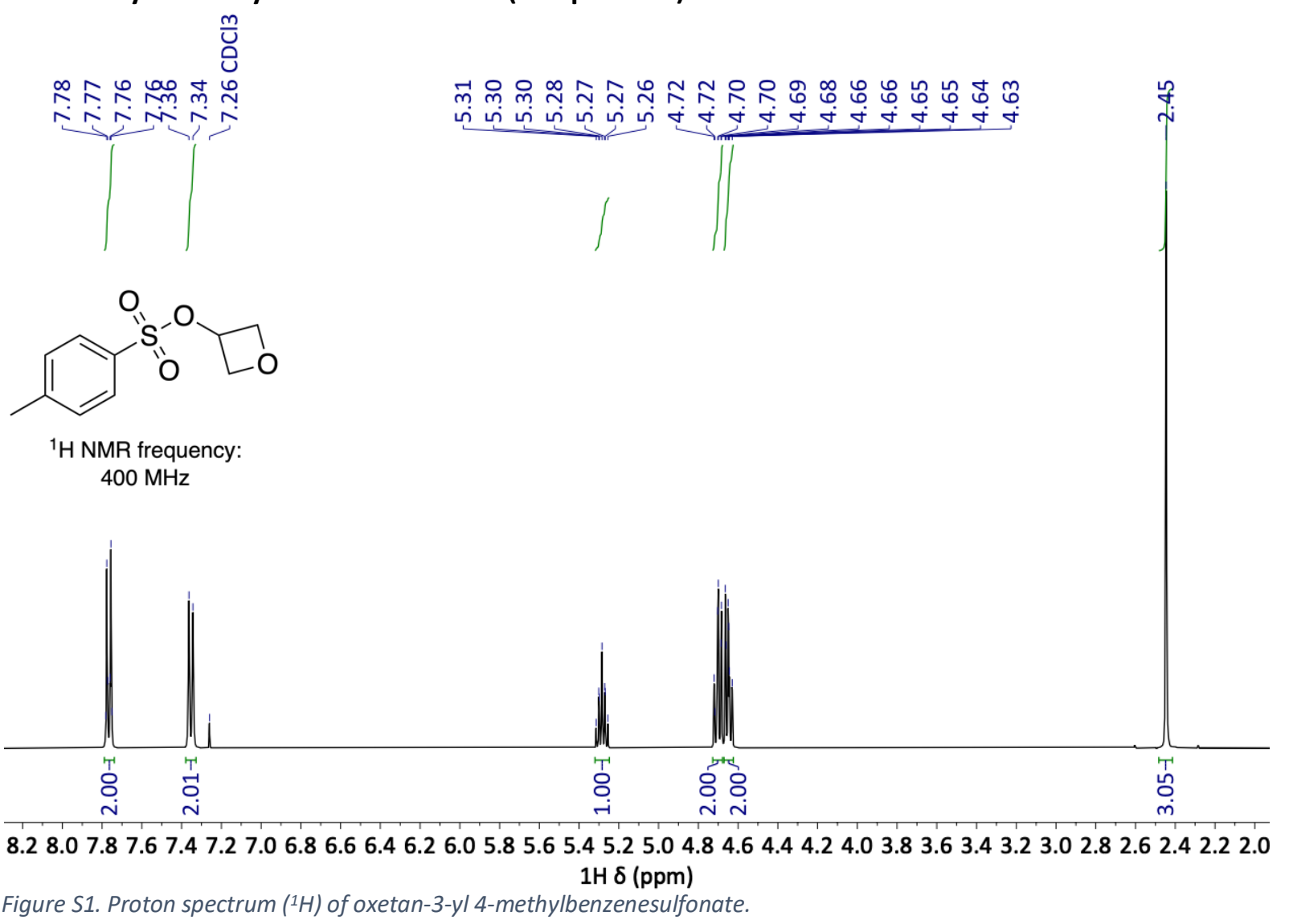




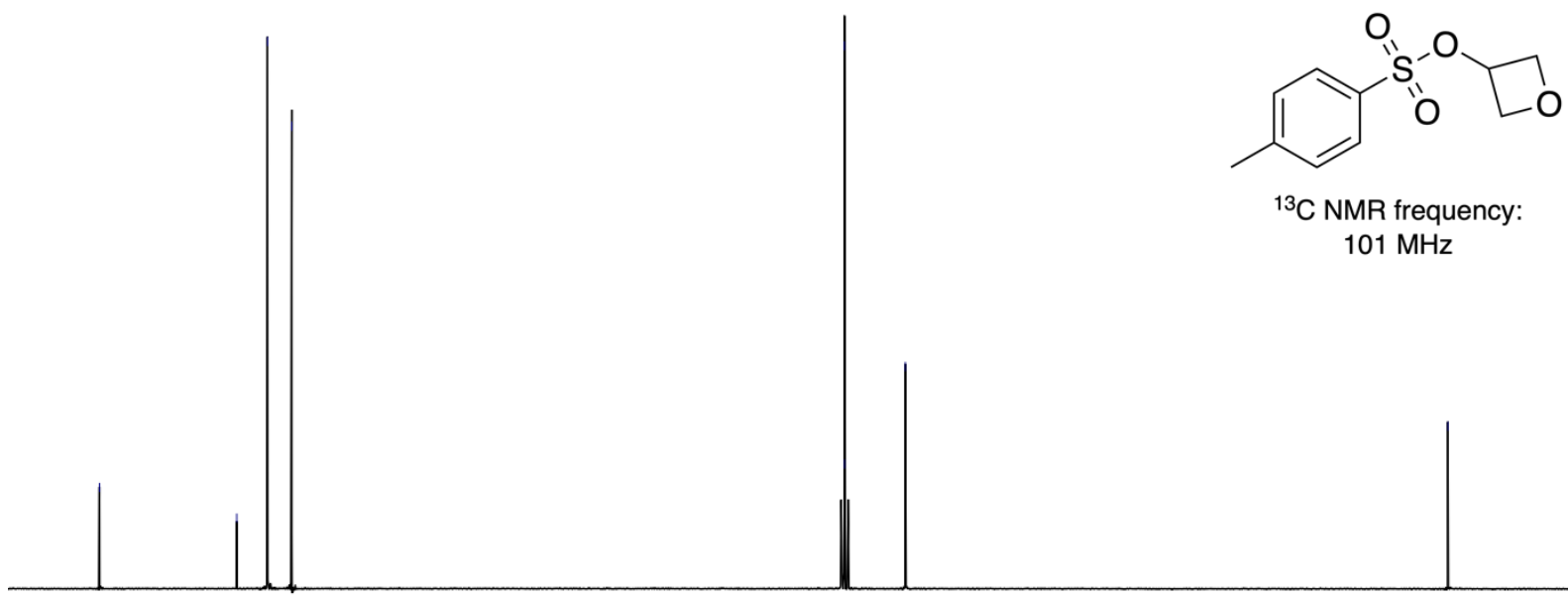

$15014514013513012512011511010510095 \quad 90 \quad 85 \quad 80 \quad 75 \quad 70 \quad 65 \quad 60 \quad 55 \quad 50 \quad 45 \quad 40 \quad 35 \quad 30 \quad 25 \quad 20 \quad 15$ $13 \mathrm{C} \delta(\mathrm{ppm})$

Figure S2. Carbon spectrum $\left({ }^{13} \mathrm{C}\right)$ of oxetan-3-yl 4-methylbenzenesulfonate.

\section{Oxetan-3-yl methanesulfonate (compound 2)}

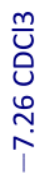
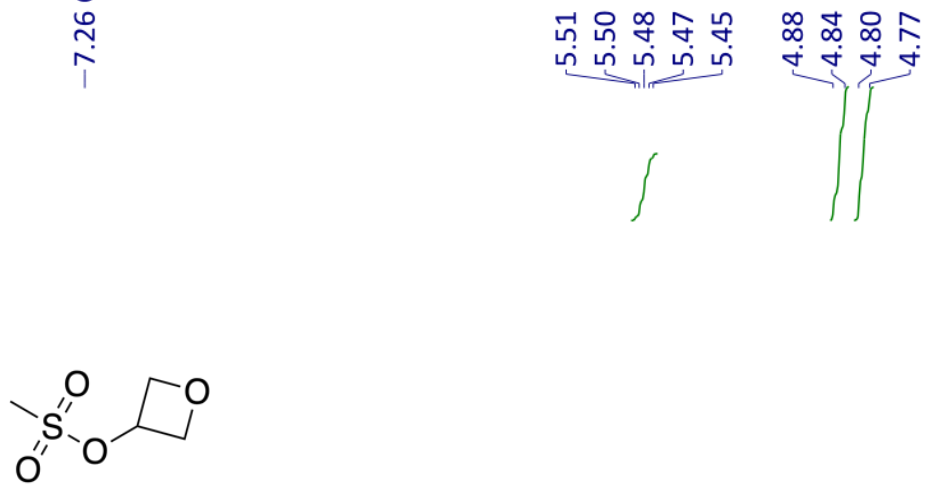

${ }^{1} \mathrm{H}$ NMR frequency:

$400 \mathrm{MHz}$

$\Phi$ 


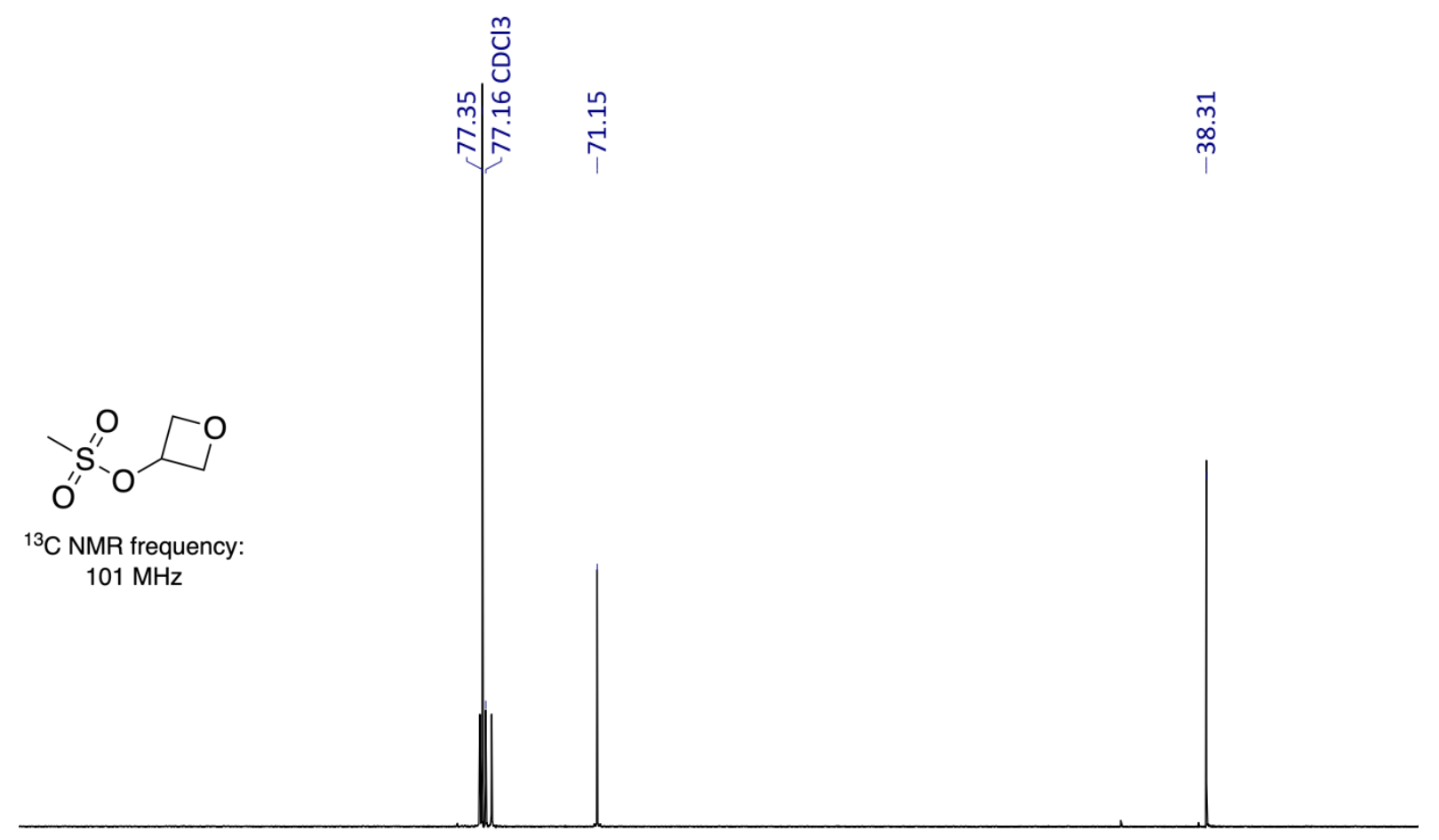

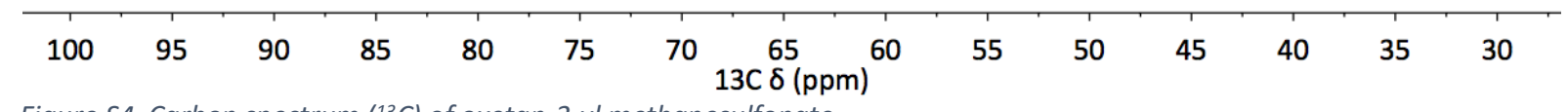

Figure S4. Carbon spectrum $\left({ }^{13} \mathrm{C}\right)$ of oxetan-3-yl methanesulfonate.

\section{3-Azidooxetan (compound 3)}

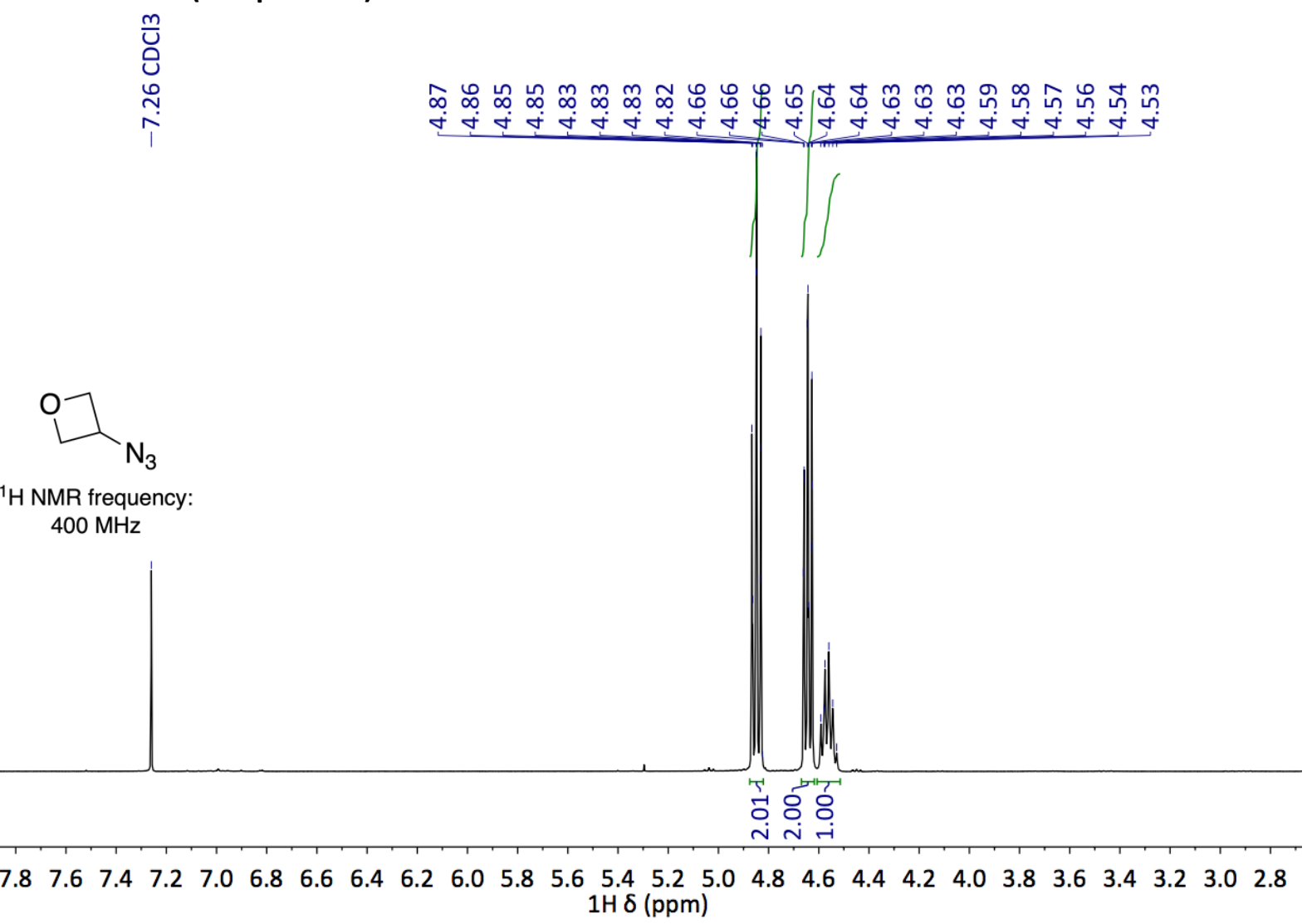
Figure S5. Proton spectrum $\left({ }^{1} \mathrm{H}\right)$ of 3-azidooxetane. 


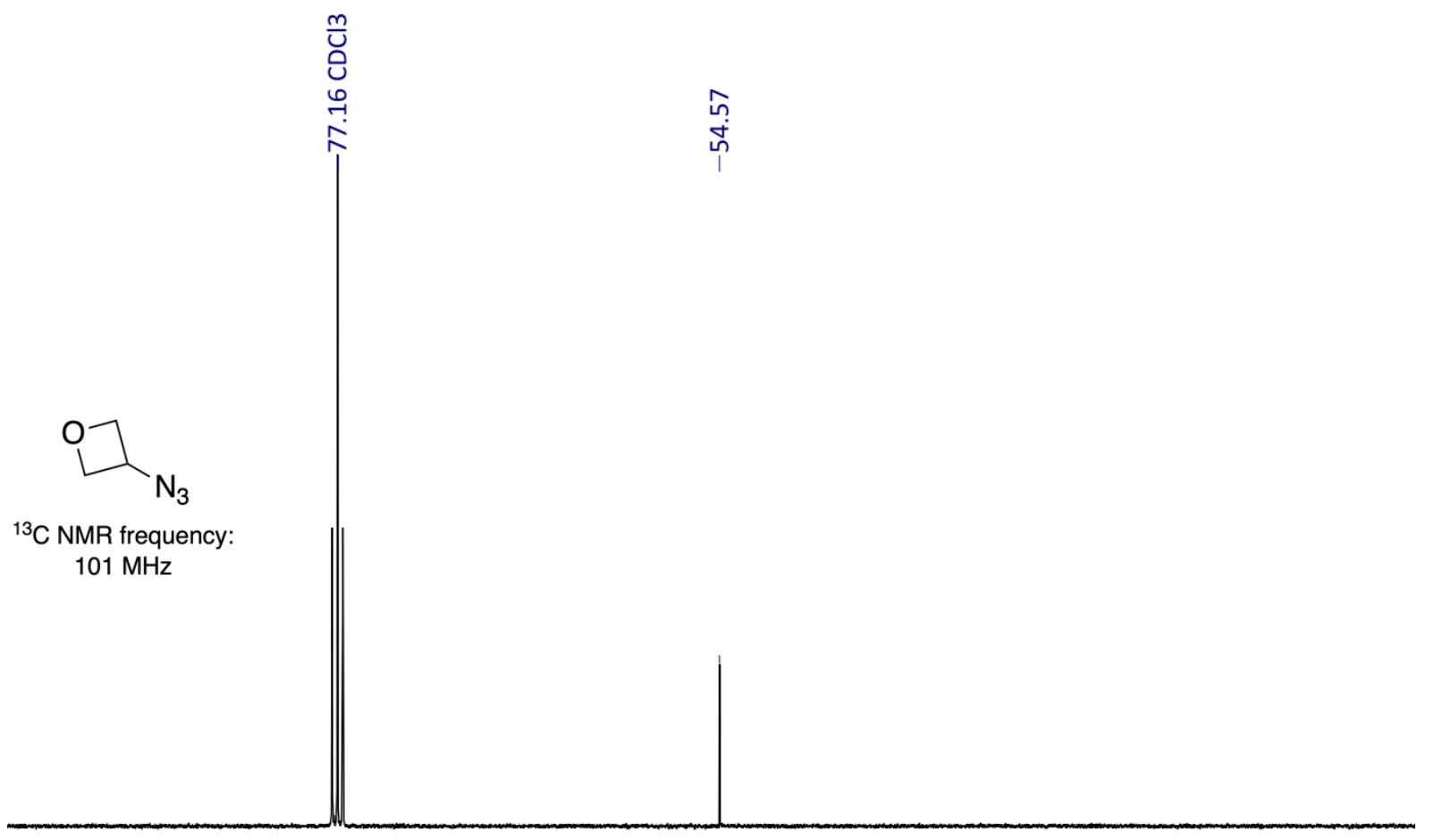

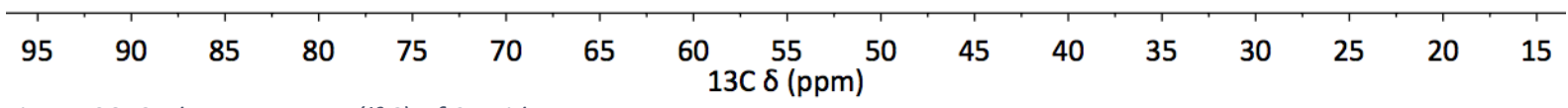

Figure S6. Carbon spectrum $\left({ }^{13} \mathrm{C}\right)$ of 3-azidooxetane.

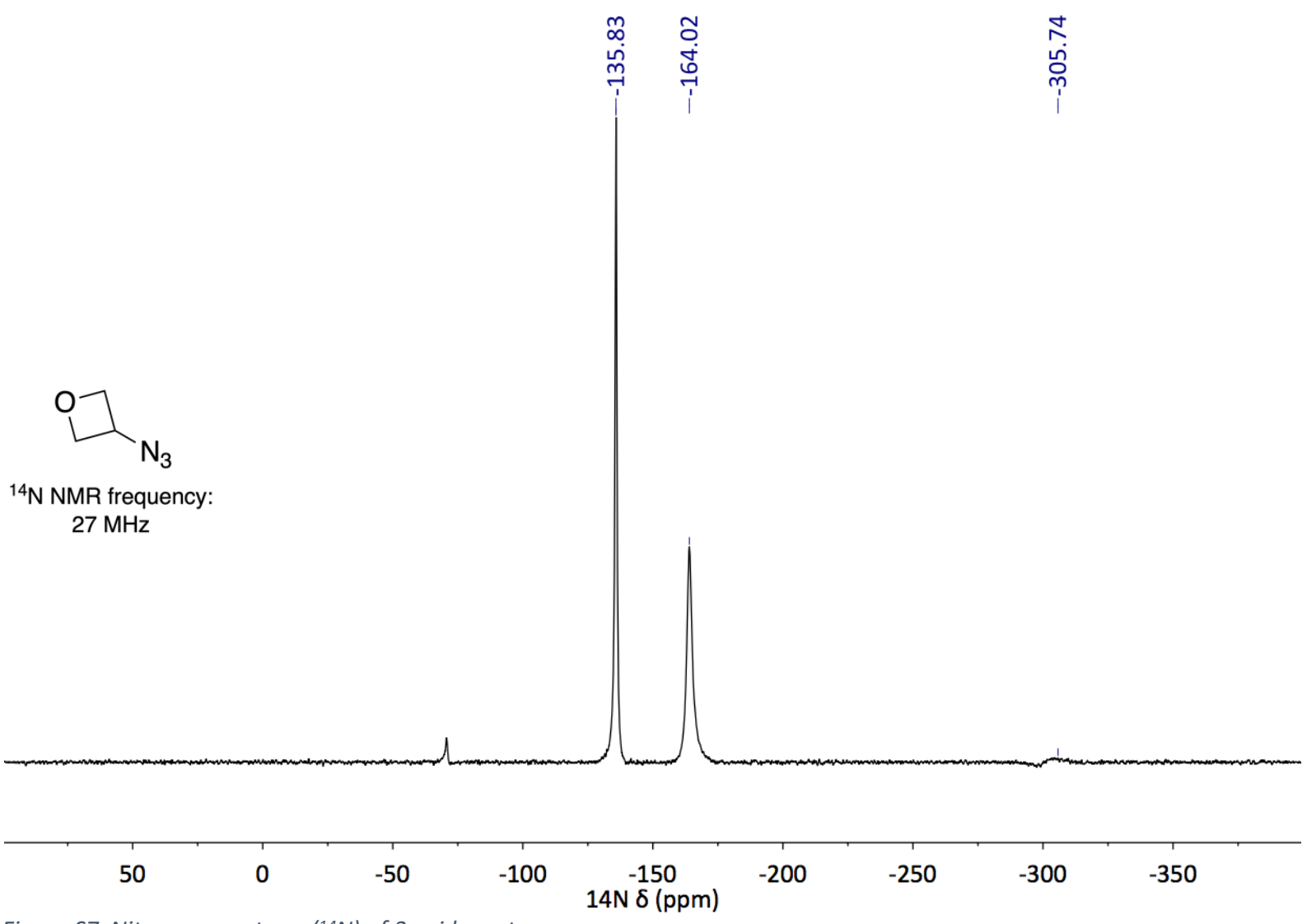

Figure S7. Nitrogen spectrum $\left({ }^{14} \mathrm{~N}\right)$ of 3-azidooxetane. 


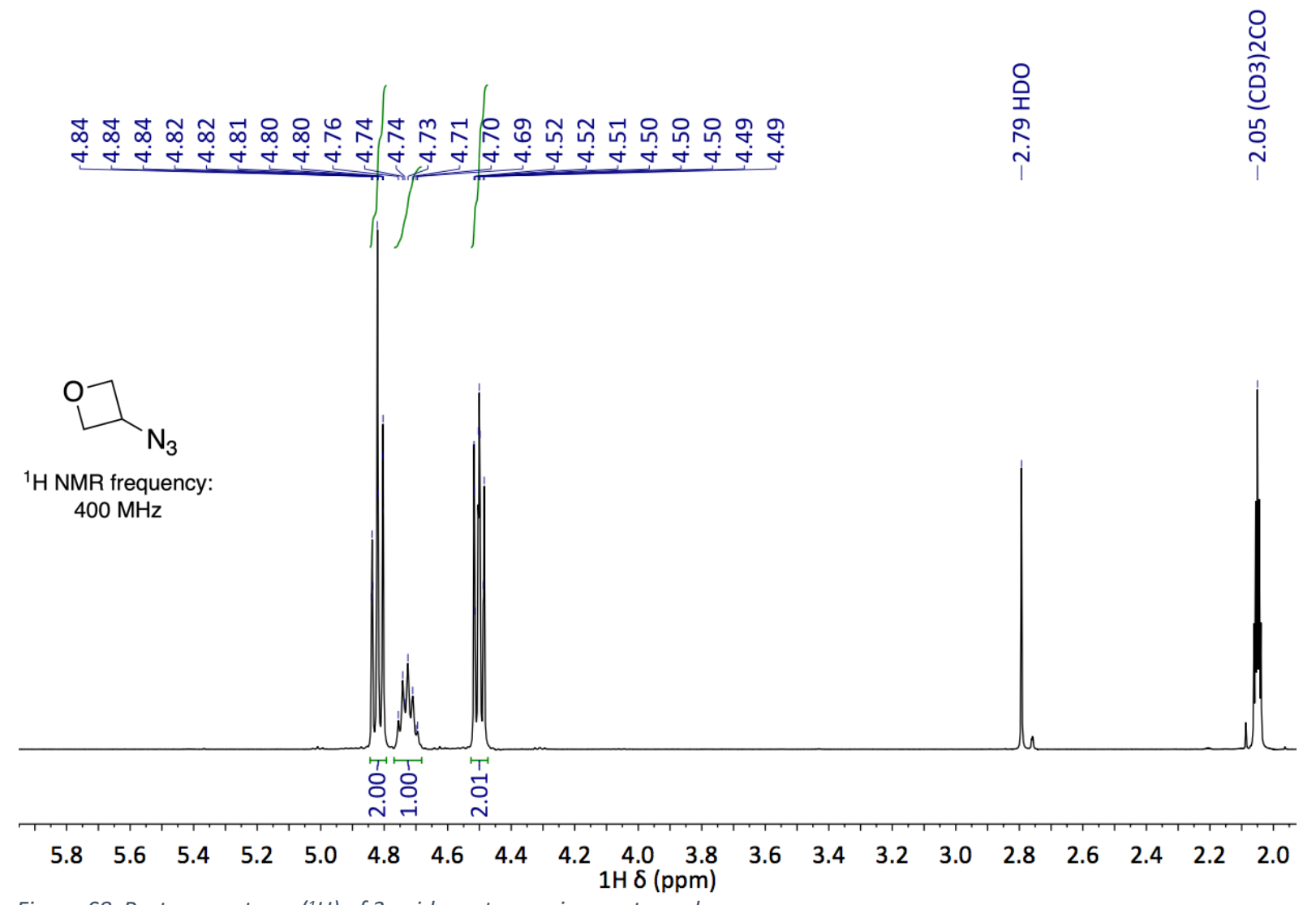

Figure S8. Proton spectrum $\left({ }^{1} H\right)$ of 3-azidooxetane using acetone- $d_{6}$.

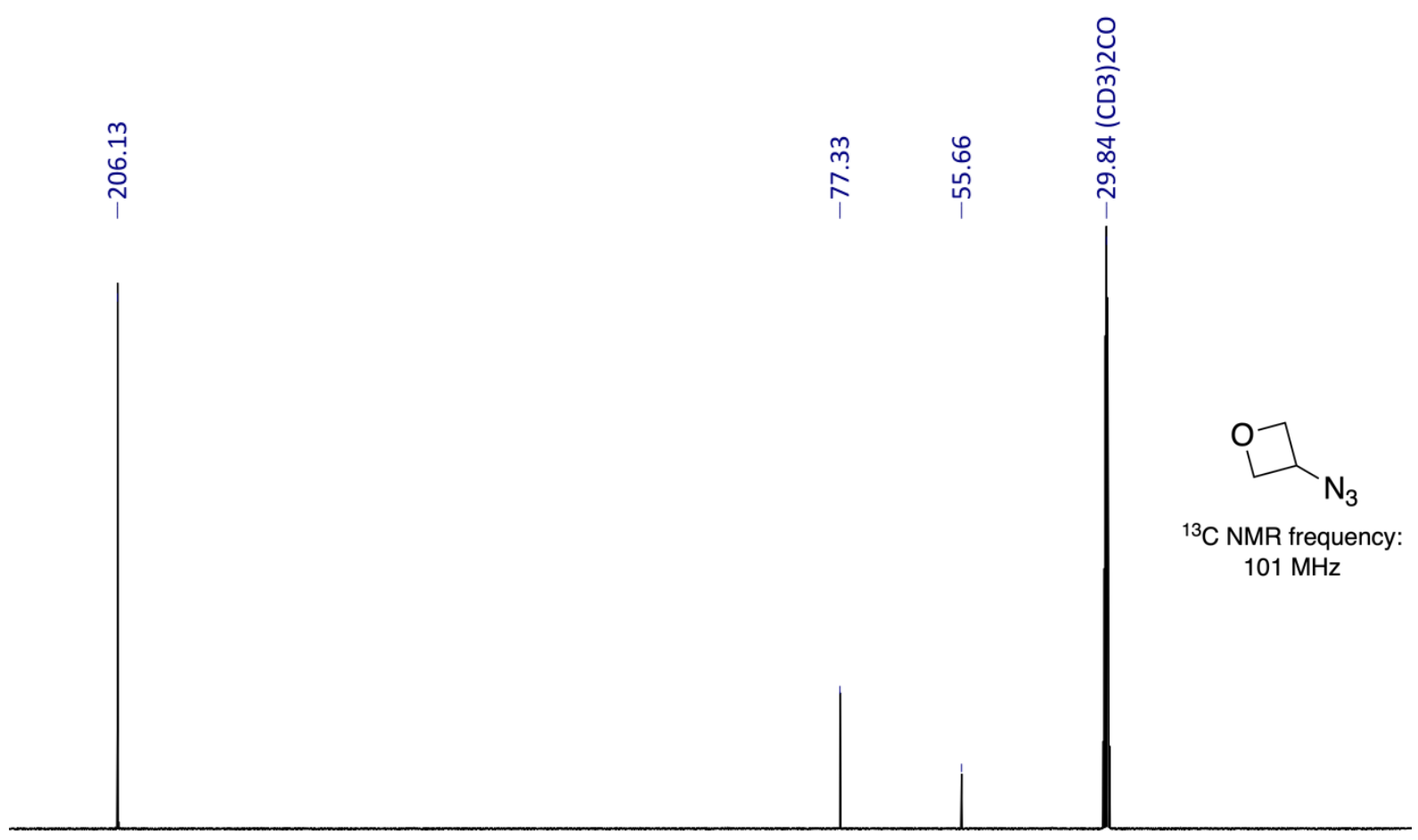

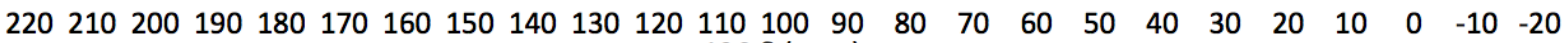
Figure 59. Carbon spectrum $\left({ }^{13} \mathrm{C}\right)$ of 3-azidooxetane using acetone-d 


\section{Glycidyl azide}

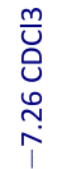

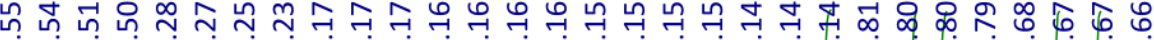

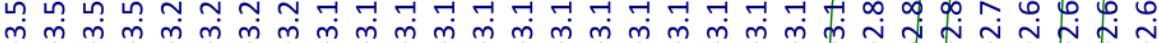

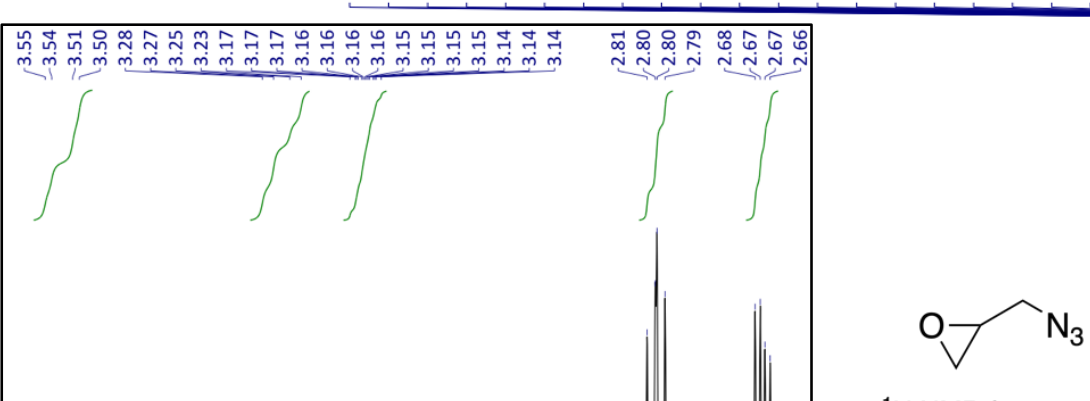

${ }^{1} \mathrm{H}$ NMR frequency: $400 \mathrm{MHz}$
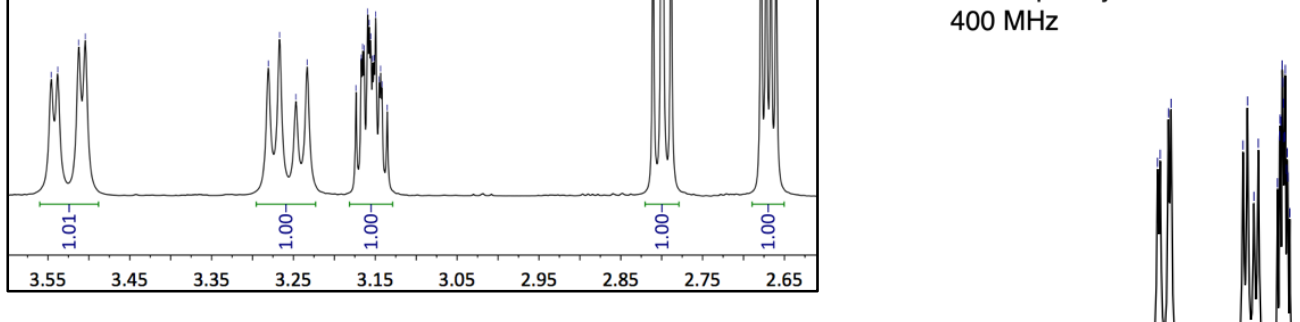

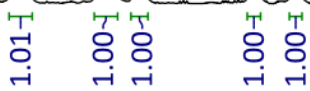

$\begin{array}{llllllllllllllllllllllll}7.2 & 7.0 & 6.8 & 6.6 & 6.4 & 6.2 & 6.0 & 5.8 & 5.6 & 5.4 & 5.2 & 5.0 & 4.8 & 4.6 & 4.4 & 4.2 & 4.0 & 3.8 & 3.6 & 3.4 & 3.2 & 3.0 & 2.8 & 2.6\end{array}$ Figure S10. Proton spectrum $\left({ }^{1} H\right)$ of glycidyl azide.

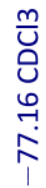
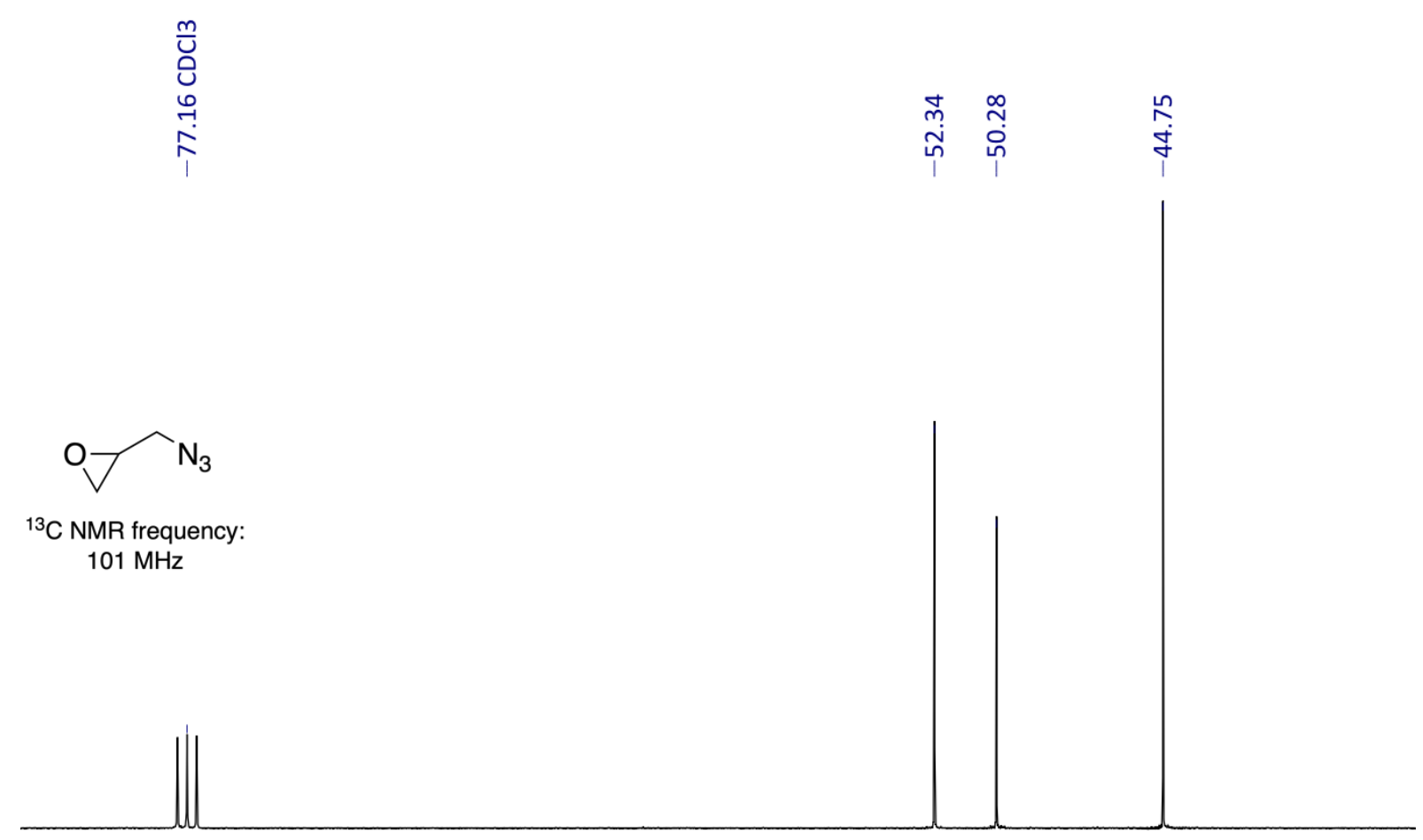

${ }^{13} \mathrm{C}$ NMR frequency

$101 \mathrm{MHz}$ $1 \mathrm{H} \delta(\mathrm{ppm})$

$\begin{array}{lllllllllllllllllllllll}82 & 80 & 78 & 76 & 74 & 72 & 70 & 68 & 66 & 64 & 62 & 60 & 58 & 56 & 54 & 52 & 50 & 48 & 46 & 44 & 42 & 40 & 38\end{array}$

Figure S11. Carbon spectrum $\left({ }^{13} \mathrm{C}\right)$ of glycidyl azide. 


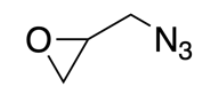

${ }^{14} \mathrm{~N}$ NMR frequency: $27 \mathrm{MHz}$

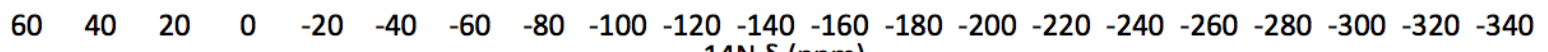

Figure S12. Nitrogen spectrum $\left({ }^{14} \mathrm{~N}\right)$ of glycidyl azide. $14 \mathrm{~N} \delta(\mathrm{ppm})$

\section{Poly(3-azidooxetane) (compound 4)}

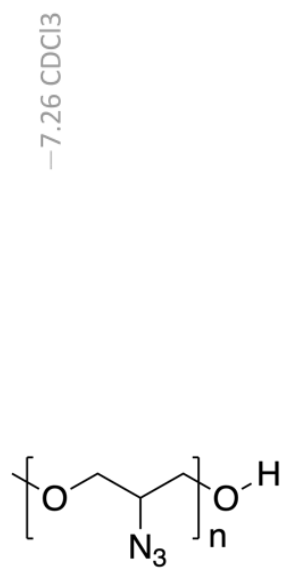

${ }^{1} \mathrm{H}$ NMR frequency: $400 \mathrm{MHz}$
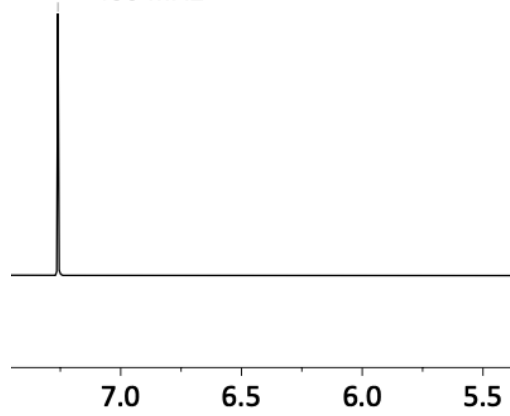

Figure S13. Proton spectrum $\left({ }^{1} \mathrm{H}\right)$ of poly(3-azidooxetane).
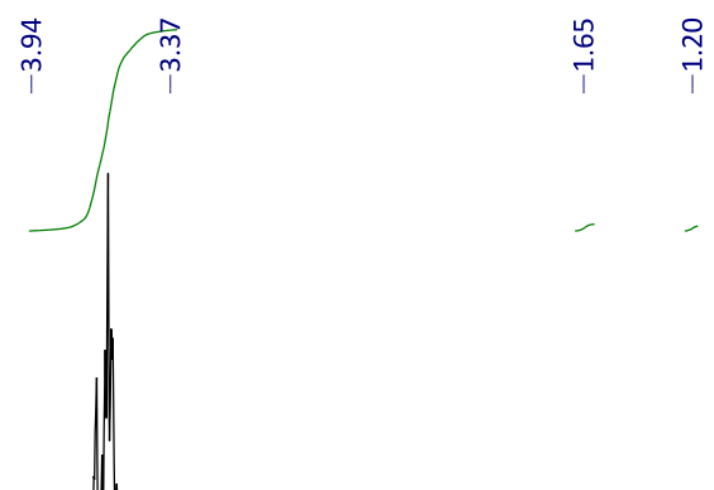
<smiles>COCC([N])CO</smiles>

${ }^{1} \mathrm{H}$ NMR frequency: $400 \mathrm{MHz}$

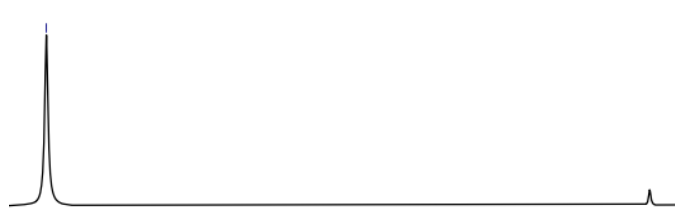

$\begin{array}{lllllllllllllllllllllllllllll}8.0 & 7.8 & 7.6 & 7.4 & 7.2 & 7.0 & 6.8 & 6.6 & 6.4 & 6.2 & 6.0 & 5.8 & 5.6 & 5.4 & 5.2 & 5.0 & 4.8 & 4.6 & 4.4 & 4.2 & 4.0 & 3.8 & 3.6 & 3.4 & 3.2 & 3.0 & 2.8 & 2.6 & 2.4\end{array}$ $1 \mathrm{H} \delta(\mathrm{ppm})$

Figure S14. Proton spectrum $\left({ }^{1} \mathrm{H}\right)$ of the attempted preparation of poly(3-azidooxetane) using triisobutylaluminum-water catalyst. DMF as co-solvent.<smiles>CCOCC(N)COC</smiles>

${ }^{1} \mathrm{H}$ NMR frequency:

$400 \mathrm{MHz}$
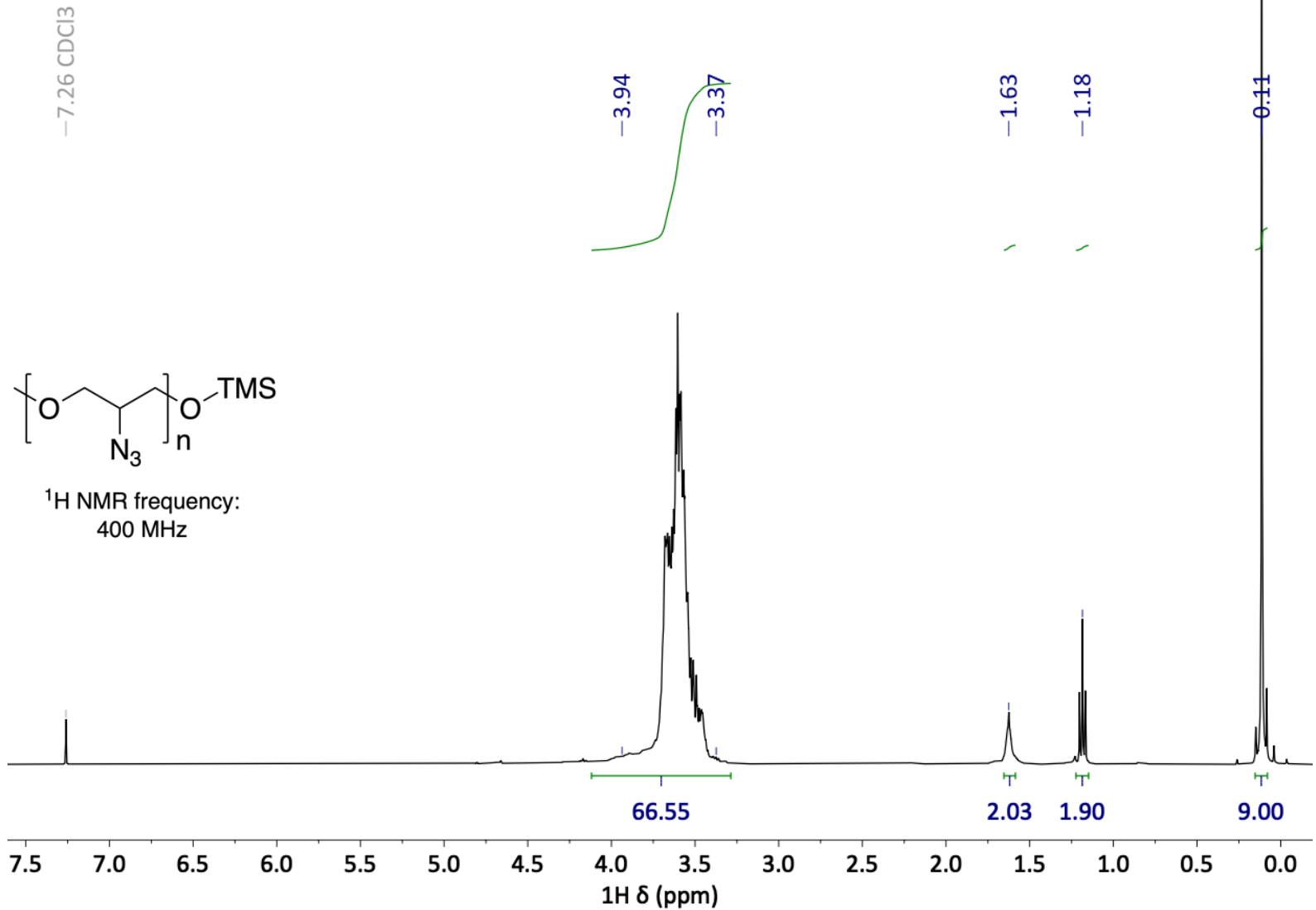

Figure S15. Proton spectrum $\left({ }^{1} \mathrm{H}\right)$ of poly(3-azidooxetane) with end-group protection (TMS) to calculate the equivalent weight. 


\section{Crystallography and Hirshfeld Analysis}

\subsection{Crystallography}

Oxetan-3-yl 4-methylbenzenesulfonate (1) and oxetan-3-yl methanesulfonate (2) were dissolved in chloroform and single crystals have been obtained after slow evaporation of the solvent. The data collection was performed using an Oxford Xcalibur3 diffractometer featuring a CCD area detector equipped with a multilayer monochromator, Photon 2 detector and a rotating-anode generator operating with Mo-K $\alpha$ radiation $(\lambda=0.7107 \AA)$. Both data collection and reduction were performed using the CrysalisPRO software. ${ }^{1}$ The structures were solved by direct methods (SIR-92, SIR-97) ${ }^{2,3}$ and refined by full-matrix least-squares on F2 (ShelXL). ${ }^{4-6}$ and finally checked using the Platon software integrated in the WinGX suite. ${ }^{7,8}$ Non-hydrogen atoms were refined anisotropically and the hydrogen atoms were located and freely refined. All structure plots depict thermal ellipsoids at the $50 \%$ probability level and hydrogen atoms are shown as small spheres of arbitrary radius.

Table S4. Detailed crystallographic information of oxetan-3-yl 4-methylbenzenesulfonate (1) and oxetan-3-yl methanesulfonate (2).

\begin{tabular}{|c|c|c|}
\hline & 1 & 2 \\
\hline Formula & $\mathrm{C}_{10} \mathrm{H}_{12} \mathrm{O}_{4} \mathrm{~S}$ & $\mathrm{C}_{4} \mathrm{H}_{8} \mathrm{O}_{4} \mathrm{~S}$ \\
\hline $\mathrm{FW}\left[\mathrm{g} \mathrm{mol}^{-1}\right]$ & 228.26 & 152.15 \\
\hline Crystal System & Monoclinic & Triclinic \\
\hline Space Group & $\mathrm{P} 21 / \mathrm{n}$ & P-1 \\
\hline Color / Habit & Colorless block & Colorless block \\
\hline Size [mm] & $0.43 \times 0.39 \times 0.22$ & $0.50 \times 0.50 \times 0.40$ \\
\hline $\mathrm{a}[\AA]$ & $9.638(6)$ & $7.8381(7)$ \\
\hline $\mathrm{b}[\AA]$ & $7.381(3)$ & $9.4125(9)$ \\
\hline c $[\AA]$ & $14.929(3)$ & $9.6897(9)$ \\
\hline$\alpha\left[^{\circ}\right]$ & 90 & 109.546(9) \\
\hline$\beta\left[^{\circ}\right]$ & $102.65(3)$ & $103.557(8)$ \\
\hline$\nu\left[{ }^{\circ}\right]$ & 90 & $96.702(7)$ \\
\hline$V\left[\AA^{3}\right]$ & $1036.36(7)$ & $639.87(11)$ \\
\hline Z & 4 & 4 \\
\hline$\rho_{\text {calc. }}\left[\mathrm{g} \mathrm{cm}^{-3}\right]$ & 1.463 & 1.580 \\
\hline$\mu\left[\mathrm{mm}^{-1}\right]$ & 0.303 & 0.446 \\
\hline$F(000)$ & 480 & 320 \\
\hline$\lambda_{\text {MoKa }}[\AA]$ & 0.71073 & 0.71073 \\
\hline $\mathrm{T}[\mathrm{K}]$ & 143 & 180 \\
\hline$\vartheta \min -\max \left[{ }^{\circ}\right]$ & $4.7450,28.5980$ & $4.3860,28.4470$ \\
\hline Dataset h; k; l & $-9: 12 ;-9: 8 ;-9: 8$ & $-9: 9 ;-4: 11 ;-12: 11$ \\
\hline Reflect. coll. & 2084 & 2558 \\
\hline Independ. Refl. & 1724 & 2207 \\
\hline $\mathrm{R}_{\text {int. }}$ & 0.027 & 0.017 \\
\hline Reflection obs. & 1724 & 2207 \\
\hline No. parameters & 137 & 165 \\
\hline R1 (obs.) & 0.0367 & 0.0322 \\
\hline
\end{tabular}




\begin{tabular}{lll} 
wR2 (all data) & 0.0941 & 0.0784 \\
$\mathrm{~S}$ & 1.051 & 1.058 \\
Resd. Dens. $\left[\mathrm{A}^{-3}\right]$ & $-0.352,0.266$ & $-0.338,0.229$ \\
Device Type & Oxford XCalibur3 CCD & Oxford XCalibur3 CCD \\
Solution & Sir92 & Sir97 \\
Refinement & SHELXLE & SHELXLE \\
Absorpt. Corr. & Multi-scan & Multi-scan \\
CCDC & 1916318 & 1916317 \\
\hline
\end{tabular}

Oxetan-3-yl 4-methylbenzenesulfonate (1)

a)

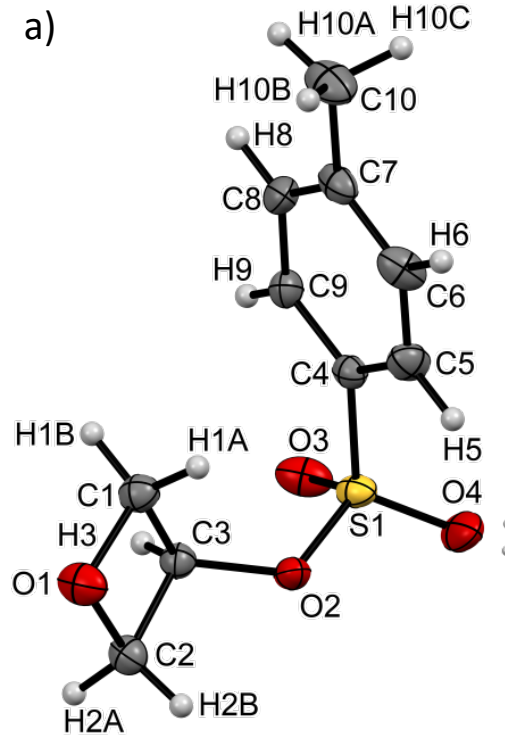

b)

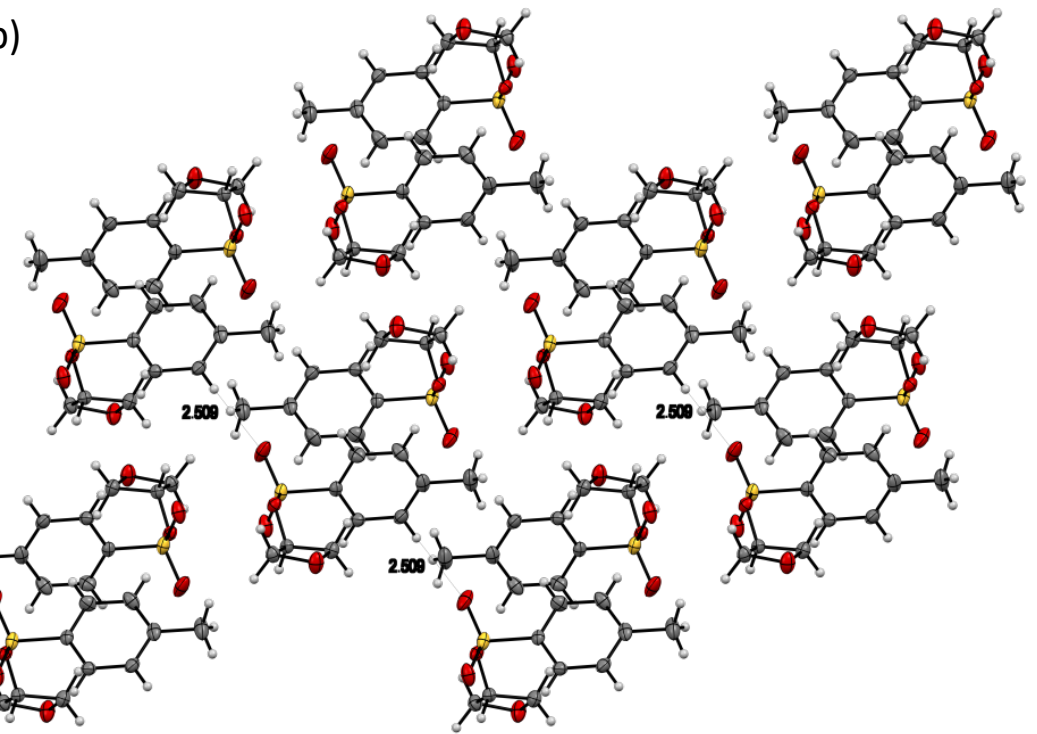

Figure S16. a) Molecular structure of compound 1. Thermal ellipsoids drawn at the 50\% probability level. b) View along $b$ axis showing the closest contact in the crystal framework.

Oxetan-3-yl 4-methanesulfonate (2)

a)

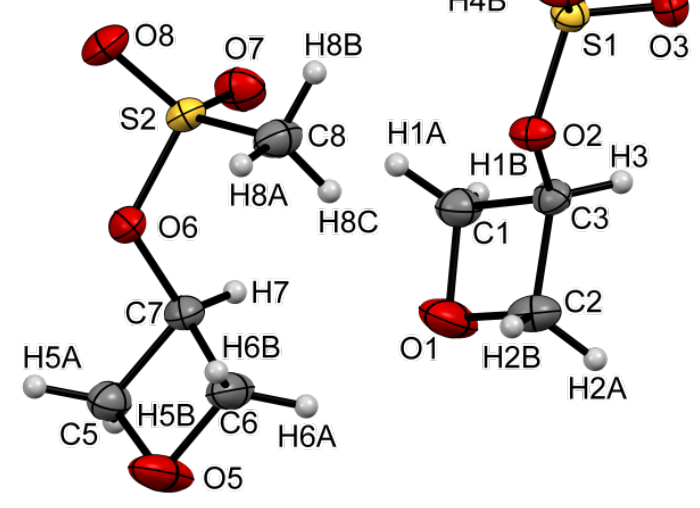

b)
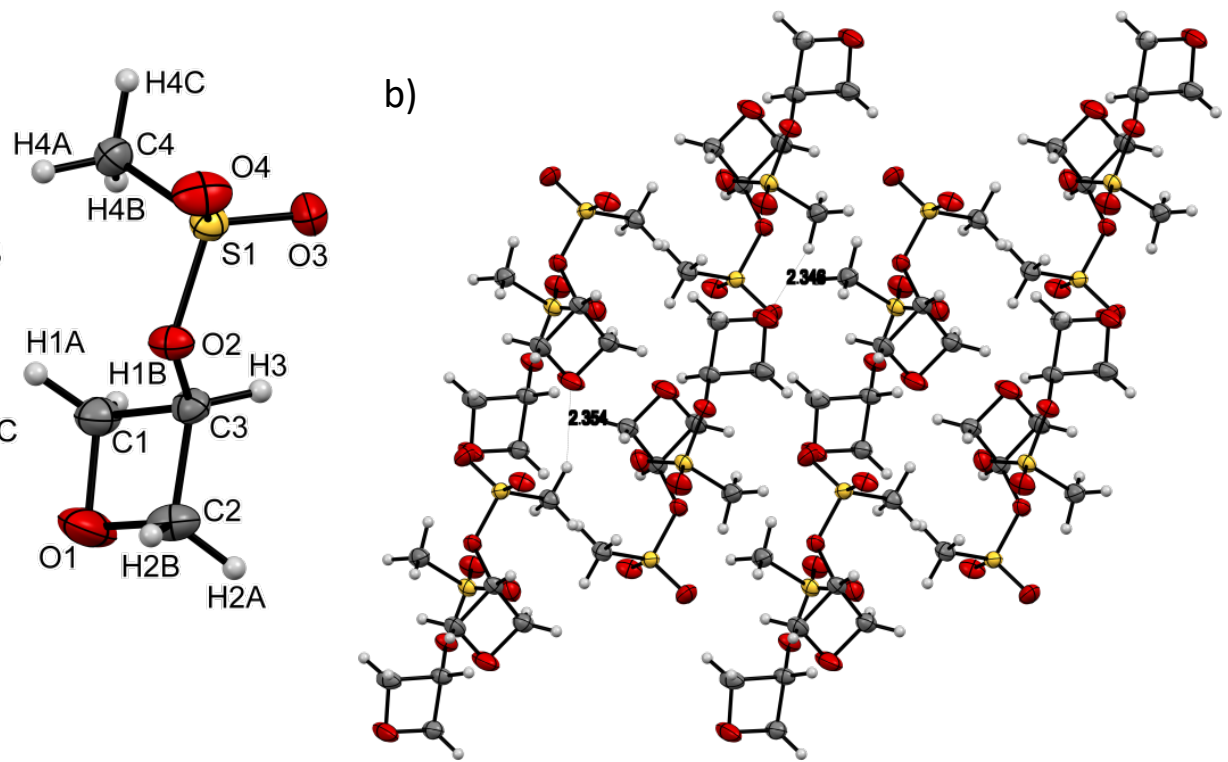

Figure S17. a) Molecular structure and crystallographic twins of compound 2. b) View along b axis showing the closest contact within the crystal. 


\section{Hirshfeld analysis:}

The following data was collected with CrystalExplorer V17.5. ${ }^{9}$
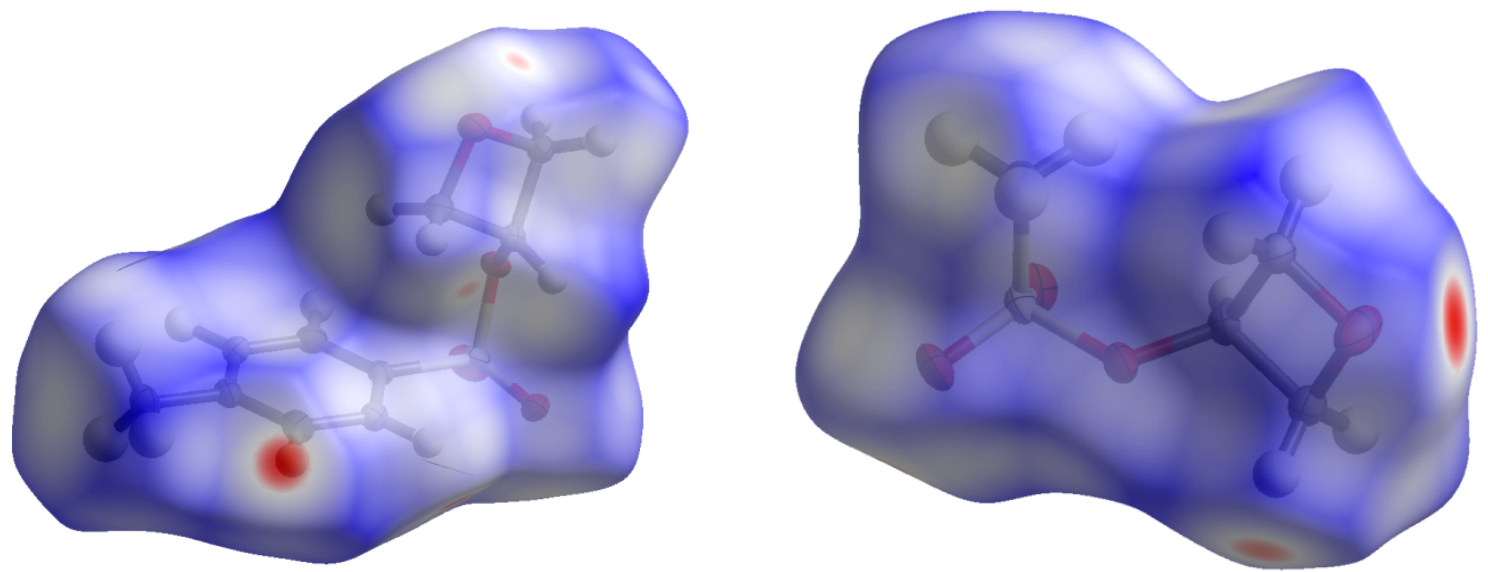

Figure S18. Calculated Hirshfeld surfaces for compound $\mathbf{1}$ (left) and compound $\mathbf{2}$ (right) indicating close contacts.
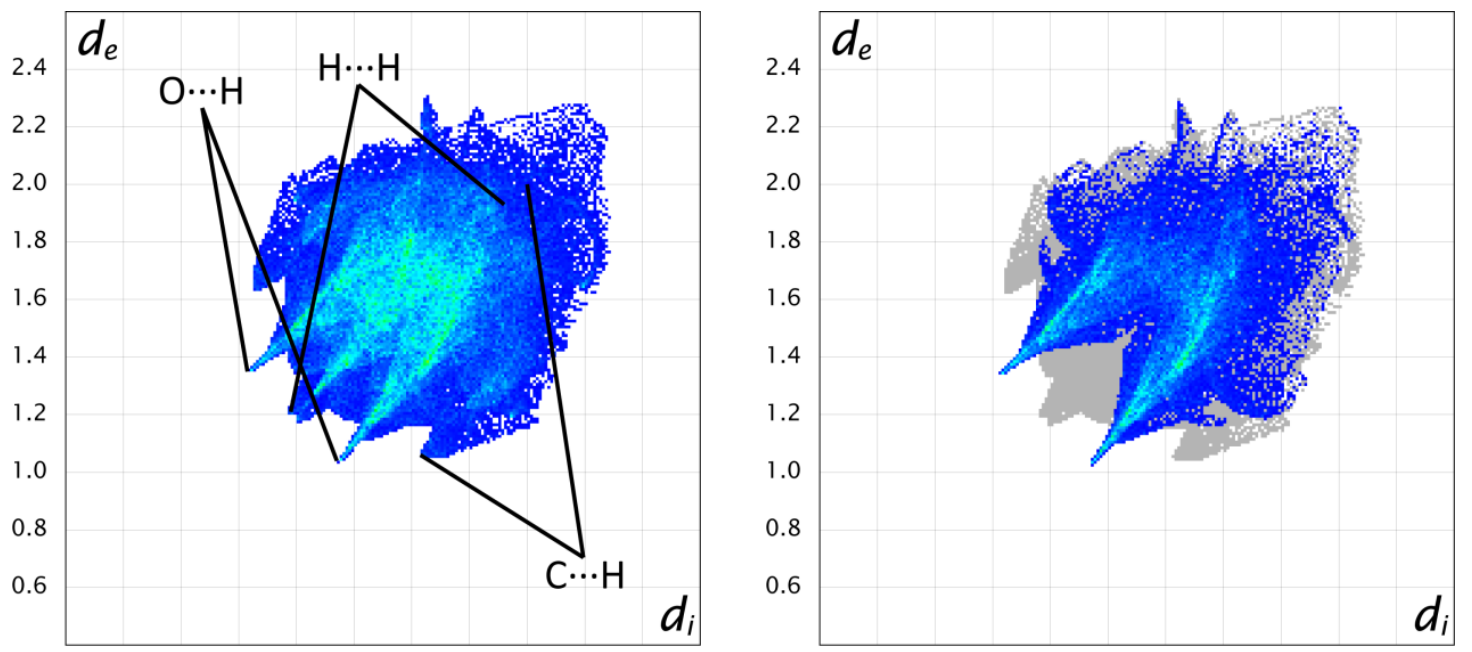

(A) $\begin{array}{llllllllllllll}0.6 & 0.8 & 1.0 & 1.2 & 1.4 & 1.6 & 1.8 & 2.0 & 2.2 & 2.4\end{array}$

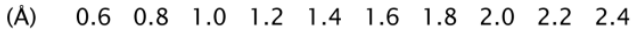
Figure S19. 2D fingerprint plot of 3-oxetanyl methylbenzenesulfonate (1) showing all interactions (left) and the high population of stabilizing $\mathrm{O}-\mathrm{H}$-interactions (right).
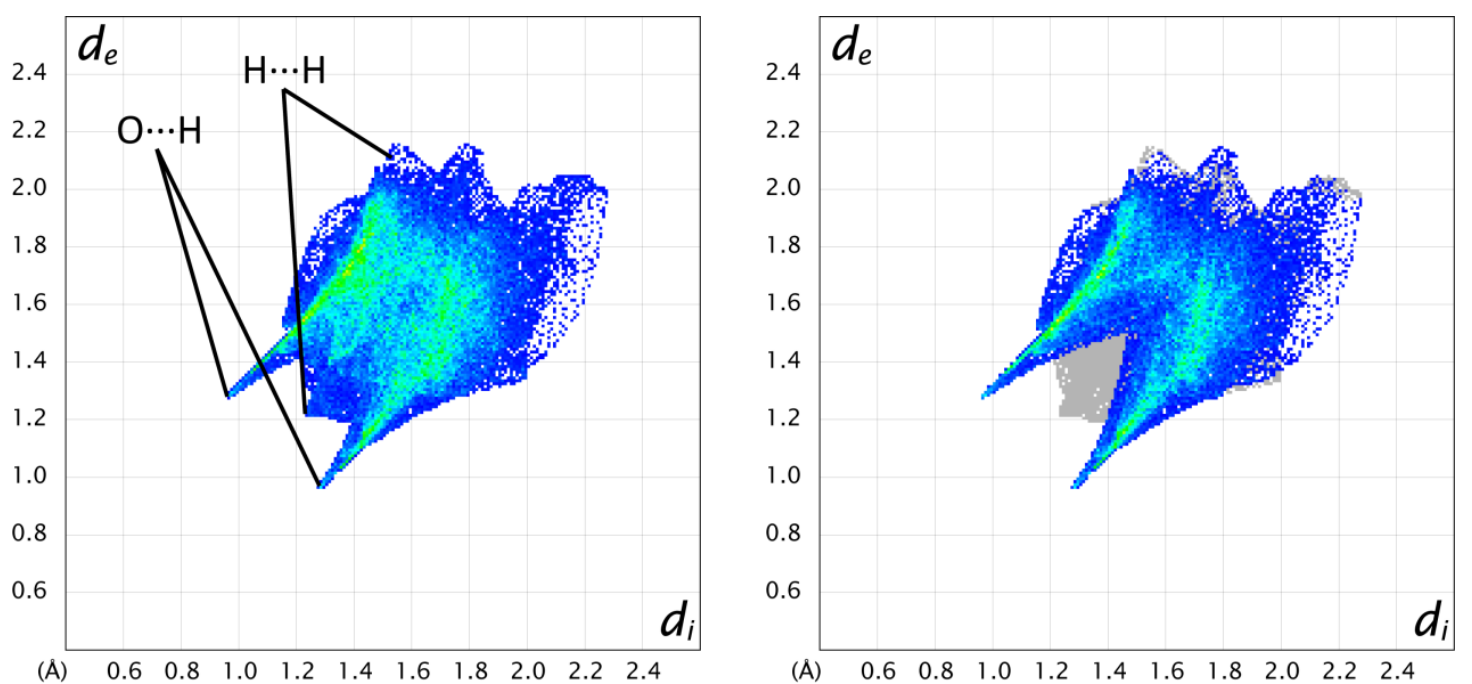

(A) $\quad \begin{array}{llllllllll}0.6 & 0.8 & 1.0 & 1.2 & 1.4 & 1.6 & 1.8 & 2.0 & 2.2 & 2.4\end{array}$ Figure S2O. 2D fingerprint plot of 3-oxetanyl methanesulfonate (2) showing all interactions (left) and the high percentage of stabilizing $\mathrm{O}-\mathrm{H}$-interactions (right). 


\section{Heat of formation calculation and thermal analysis}

The atomization method was used to determine the heat of formation of 3-azidooxetane and glycidyl azide using the atom energies in Table $5 .^{10}$

$$
\Delta_{\mathrm{f}} \mathrm{H}^{\circ}{ }_{(\mathrm{g}, \mathrm{M}, 298)}=\mathrm{H}_{(\text {molecule, 298) }}-\sum \mathrm{H}^{\circ}{ }_{\text {(atoms, 298) }}+\sum \Delta_{\mathrm{f}} \mathrm{H}^{\circ}{ }_{\text {(atoms, 298) }}
$$

Table S5. CBS-4M electronic enthalpies for atoms $C, H, N$ and $O$ and their literature values.

\begin{tabular}{|l|l|l|}
\hline & $-\mathrm{H}^{298} /$ a.u. & $\Delta_{\mathrm{f}} \mathrm{H}_{\text {gas }}^{11}$ \\
\hline $\mathrm{H}$ & 0.500991 & 217.998 \\
\hline $\mathrm{C}$ & 37.786156 & 716.68 \\
\hline $\mathrm{N}$ & 54.522462 & 472.68 \\
\hline $\mathrm{O}$ & 74.991202 & 249.18 \\
\hline
\end{tabular}

The Gaussian16 program package was used to calculate room temperature enthalpies on the CBS-4M level of theory. ${ }^{12}$ To obtain the energy of formation for the liquid phase of $\mathbf{1}$ and 2, Trouton's Rule was applied $\left(\Delta \mathrm{H}_{\mathrm{sub}}=90 \cdot \mathrm{T}_{\mathrm{m}}\right)$.

Table S6. Heat of formation calculation results for compounds 3 and glycidyl azide.

\begin{tabular}{|l|l|l|l|l|l|l|}
\hline$M$ & $-H^{298[a]}[$ a.u. $]$ & $\begin{array}{l}\Delta_{\mathrm{f}} \mathrm{H}^{\circ}(\mathrm{g}, \mathrm{M}) \\
{[\mathrm{b}]} \\
{\left[\mathrm{kJ} \mathrm{mol}^{-1}\right]}\end{array}$ & $\begin{array}{l}\Delta_{\mathrm{sub}} \mathrm{H}^{\circ}(\mathrm{M})^{[\mathrm{c}]} \\
{\left[\mathrm{kJ} \mathrm{mol}^{-1}\right]}\end{array}$ & $\begin{array}{l}\Delta_{\mathrm{f}} \mathrm{H}^{\circ}(\mathrm{s} / \mathrm{l})^{[\mathrm{d}]}[\mathrm{kJ} \\
\left.\mathrm{mol}^{-1}\right]\end{array}$ & $\begin{array}{l}\Delta \mathrm{n} \\
{\left[\mathrm{kJ} \mathrm{kg}^{-1}\right]}\end{array}$ \\
\hline 3-AO & 356.191018 & 262.7 & 41.8 & 221.0 & -4.5 & 2342.4 \\
\hline GA & 356.192618 & 258.5 & 42.0 & 216.5 & -4.5 & 2297.2 \\
\hline
\end{tabular}

[a] CBS-4M electronic enthalpy; [b] gas phase enthalpy of formation; [c] sublimation enthalpy; [d] standard liquid state enthalpy of formation; [e] solid state energy of formation.

The thermal behavior of compounds $\mathbf{1}$ and $\mathbf{2}$ was analyzed by DSC at a heating rate of $5{ }^{\circ} \mathrm{C} \mathrm{min}^{-1}$ to determine their decomposition temperatures (S20). In addition, the boiling points and decomposition temperatures for compound $\mathbf{3}$ and glycidyl azide were assed. Last but not least, the thermal behavior of poly(3-azidooxetane) (4) was assessed to determine both glass transition and decomposition temperature. The obtained thermograms are depicted.

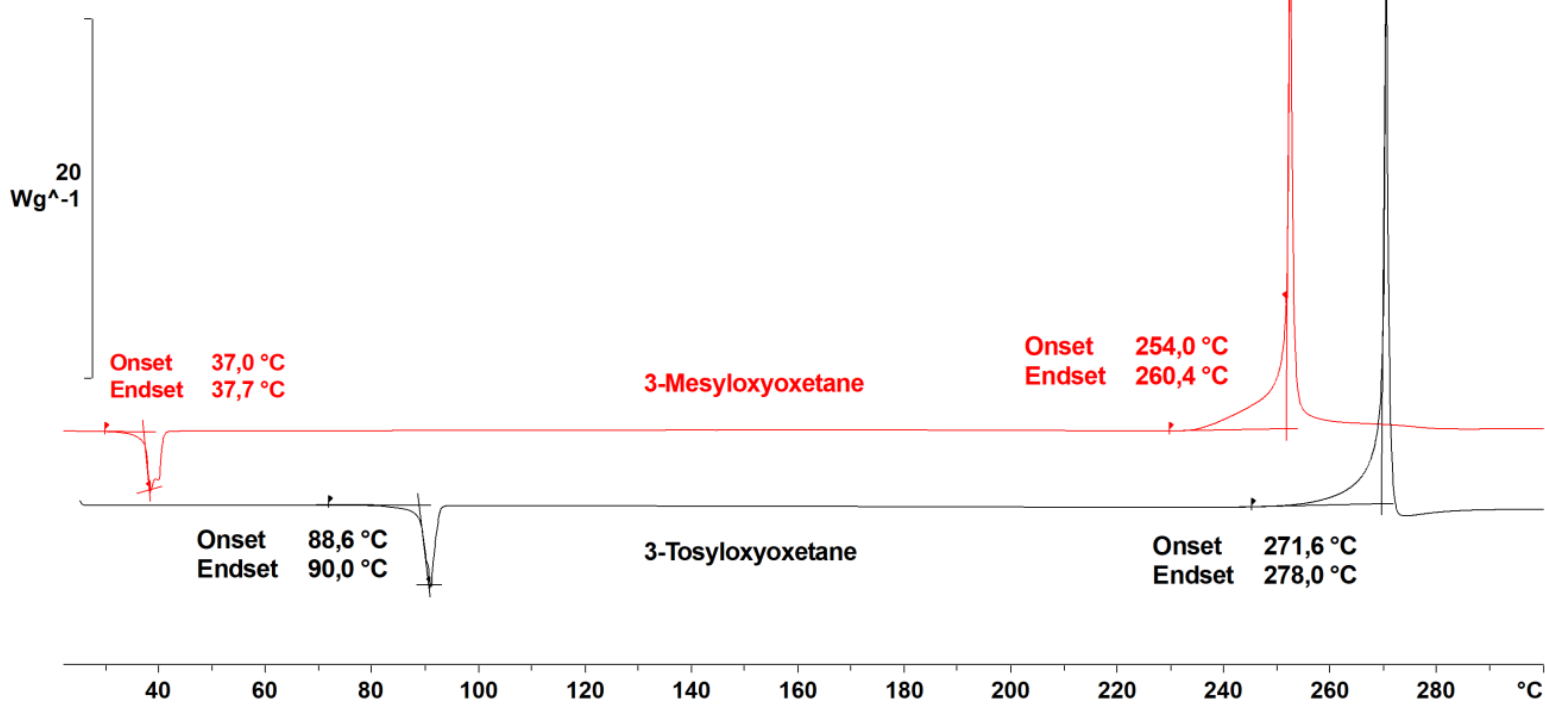

Figure S21. DSC evaluation of compounds 1 and 2. 


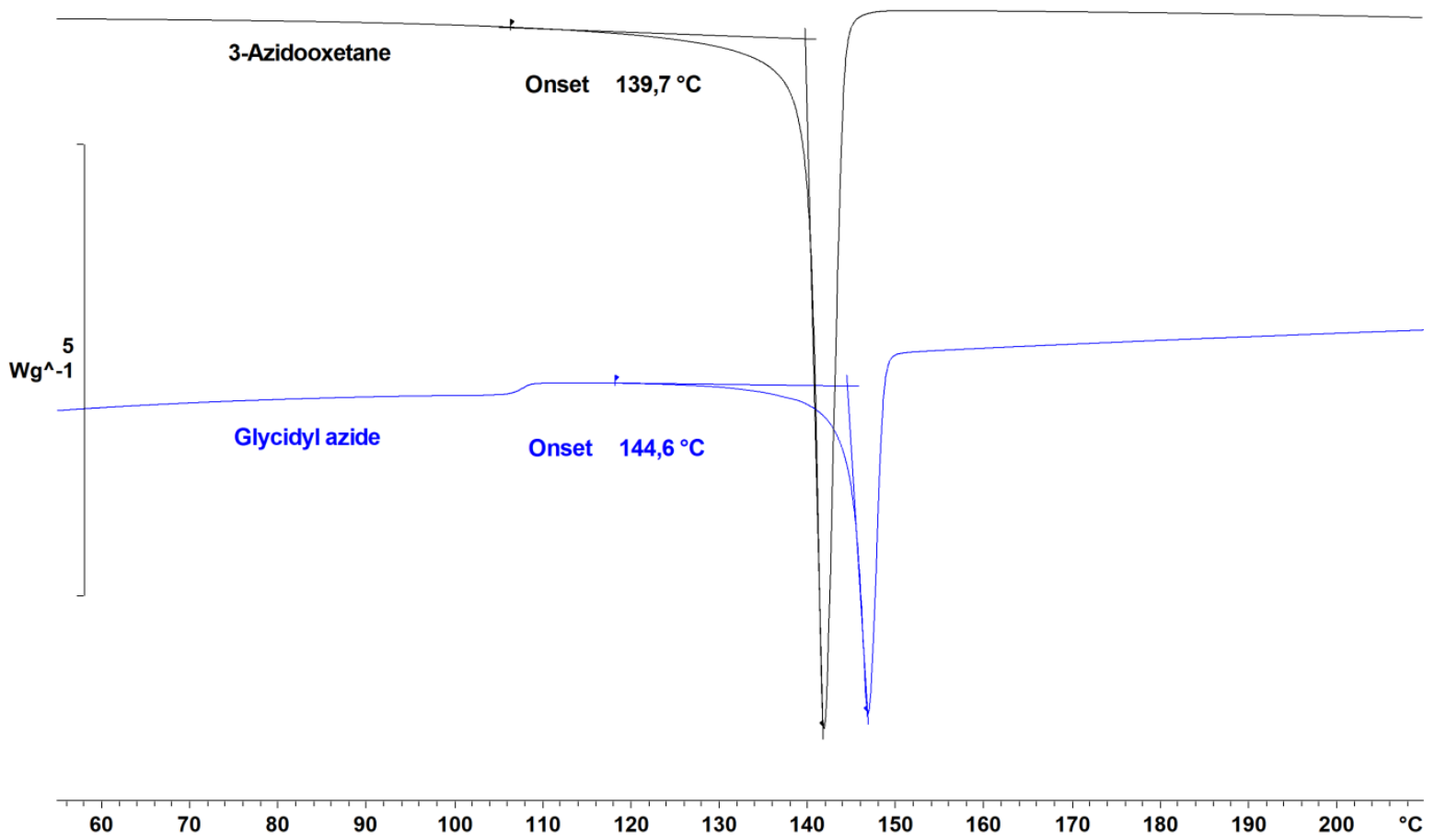

Figure S22. DSC evaluation for the boiling points of 3-azidooxetane (3) and glycidyl azide.

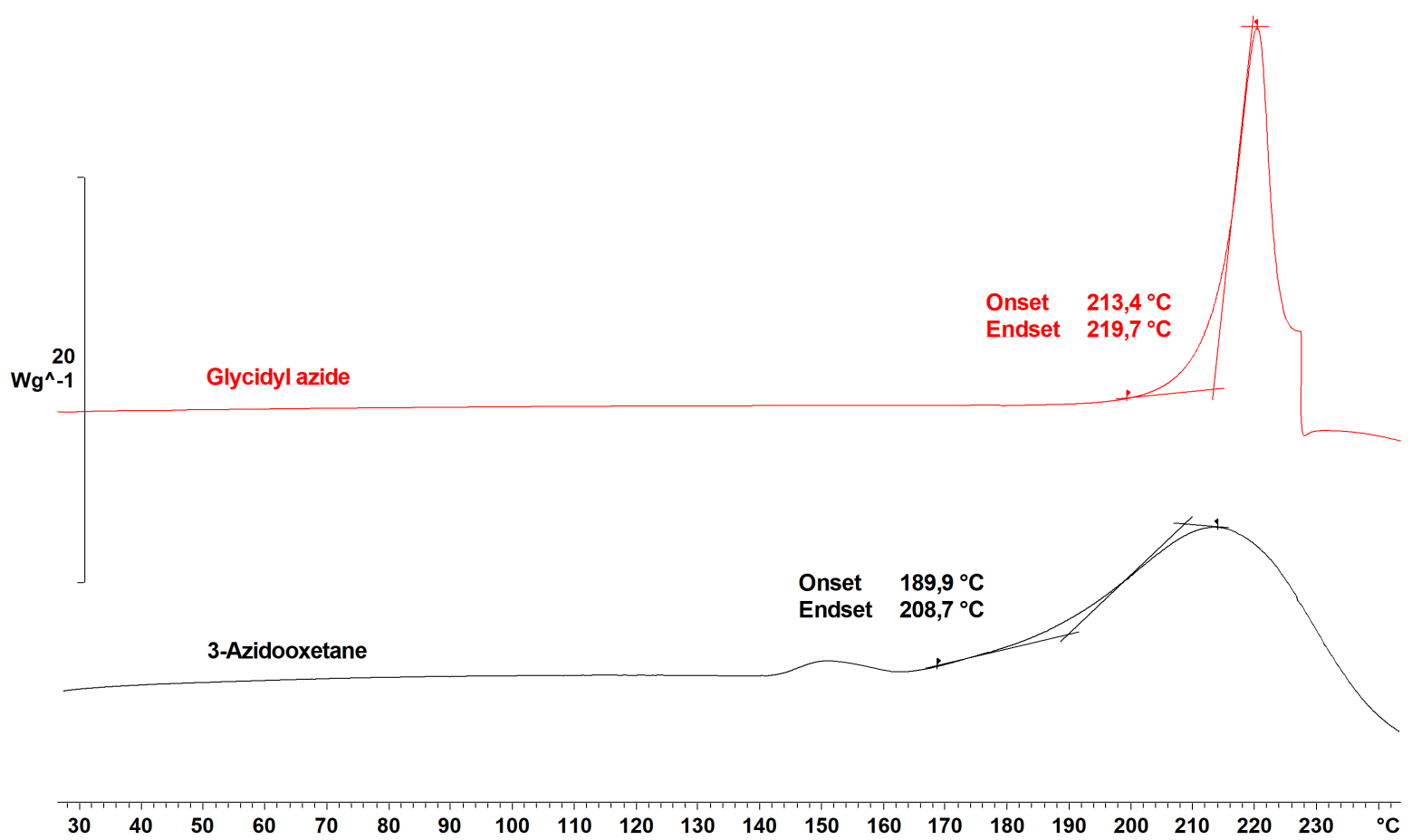

Figure S23. DSC evaluation of the decomposition temperatures of 3-azidooxetane (3) and glycidyl azide in closed 40 $\mathrm{L}$ aluminum crucibles. 


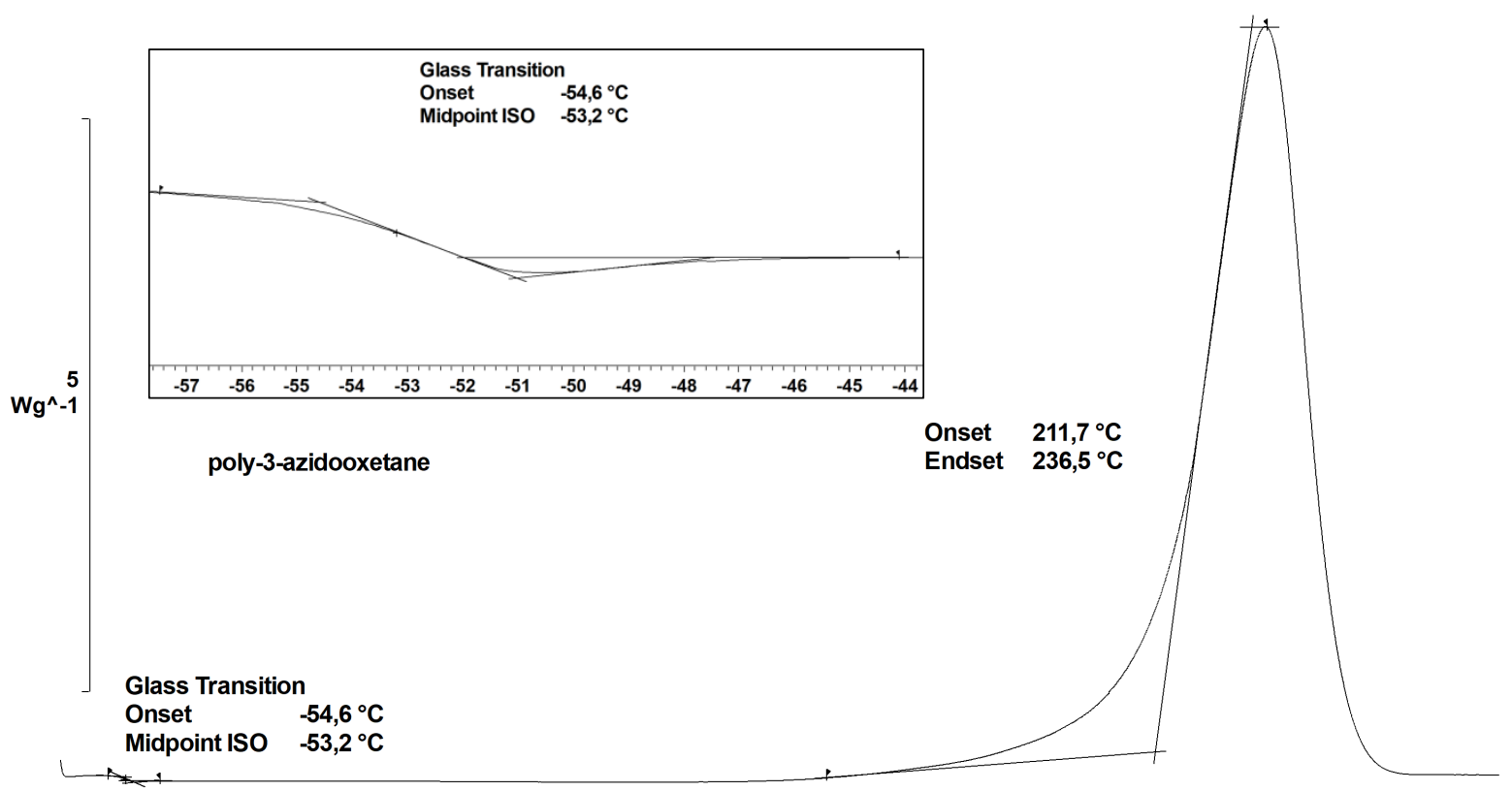

$\begin{array}{llllllllllllllllllllllll}-60 & -40 & -20 & 0 & 20 & 40 & 60 & 80 & 100 & 120 & 140 & 160 & 180 & 200 & 220 & 240 & 260 & 280 & { }^{\circ} \mathrm{C}\end{array}$ Figure S24. Thermal analysis of poly(3-azidooxtane) with regard to glass transition and decomposition temperature.

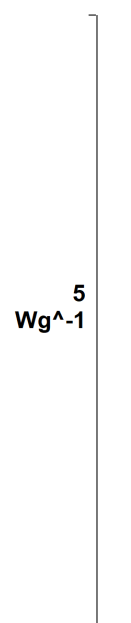

poly-3-Azidooxetane $(2.5 \mathrm{mg})$

poly-3-Azidooxetane $(5.0 \mathrm{mg}$, TIBA)

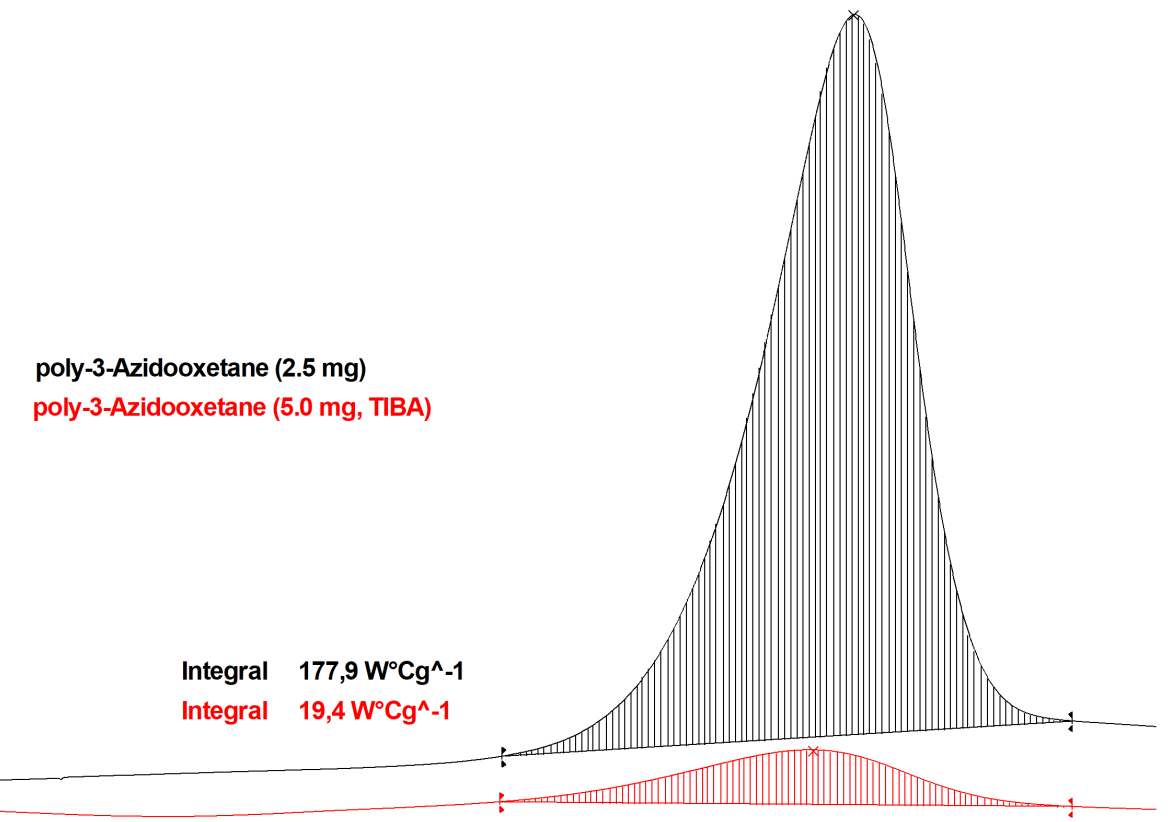

60

80

100

120

140

160

180

200

Figure S25. Thermal decomposition analysis of low-molecular-weight poly(3-azidooxtane) (black) in comparison to polym material (red) obtained with triisobutylaluminum-water catalyst (TIBA). The integrals indicate the reduced nitrogen content in the second case. 


\section{Detonation parameters and hot-plate test}

The following output data was obtained using the EXPLO5 V6.04 thermochemical code. ${ }^{13}$

\section{Glycidylazide}

$C(3,000) H(5,000) N(3,000) O(1,000)$

Molecular weight $\quad=99,09$

Density of explosive $\quad=1,14 \mathrm{~g} / \mathrm{cm} 3$

Oxygen balance $\quad=-121,0908 \%$

Enthalpy of formation $\quad=2229,22 \mathrm{~kJ} / \mathrm{kg}$

Internal energy of formation $=2341,78 \mathrm{~kJ} / \mathrm{kg}$

Detonation parameters (at the C-J point) :

$\begin{array}{lll}\text { Heat of detonation } & =-4444,648 \mathrm{~kJ} / \mathrm{kg} \\ \text { Detonation temperature } & =2793,657 \mathrm{~K} \\ \text { Detonation pressure } & =10,55701 \mathrm{GPa} \\ \text { Detonation velocity } & =6121,845 \mathrm{~m} / \mathrm{s} \\ \text { Particle velocity } & =1512,704 \mathrm{~m} / \mathrm{s} \\ \text { Sound velocity } & =4609,141 \mathrm{~m} / \mathrm{s} \\ \text { Density of products } & =1,514144 \mathrm{~g} / \mathrm{cm} 3 \\ \text { Volume of products } & =0,6604391 \mathrm{~cm} 3 / \mathrm{g} \\ \text { Exponent 'Gamma' } & =3,046954 \\ \text { Moles of gaseous products } & =3,356081 \mathrm{~mol} / \mathrm{mol} \text { explosive } \\ \text { Moles of condensed products } & =2,012745 \mathrm{~mol} / \mathrm{mol} \text { explosive } \\ \text { Volume of gas at STP } & =828,2757 \mathrm{dm} 3 / \mathrm{kg} \\ \text { Mean molecular mass of gas. prod. } & =22,3226 \mathrm{~g} / \mathrm{mol} \\ \text { Mean molecular mass of cond.prod. } & =12,011 \mathrm{~g} / \mathrm{mol} \\ \text { Mean molecular mass of all prod. } & =18,45683 \mathrm{~g} / \mathrm{mol} \\ \text { Entropy of products } & =7,745953 \mathrm{~kJ} / \mathrm{kg} \mathrm{K} \\ \text { Internal energy of products } & =5588,796 \mathrm{~kJ} / \mathrm{kg}, \text { i.e. } 6,371227 \mathrm{~kJ} / \mathrm{cm} 3 \\ \text { Compression energy } & =1144,148 \mathrm{~kJ} / \mathrm{kg}, \text { i.e. } 1,304328 \mathrm{~kJ} / \mathrm{cm} 3 \\ \text { Total heat energy } & =-4444,648 \mathrm{~kJ} / \mathrm{kg}, \text { i.e. }-5,066899 \mathrm{~kJ} / \mathrm{cm} 3\end{array}$

Composition of detonation products:

\begin{tabular}{llll} 
Products & $\mathrm{mol} / \mathrm{mol}$ & $\mathrm{mol} / \mathrm{kg}$ & $\mathrm{Mol} \%$ \\
\hline $\mathrm{C}(\mathrm{gr})=$ & 2,012745 & 20,31168 & 37,48948 \\
$\mathrm{~N} 2=$ & 1,368801 & 13,8133 & 25,49535 \\
$\mathrm{H} 2 \mathrm{O}=$ & 0,6685275 & 6,746466 & 12,45202 \\
$\mathrm{CH} 4=$ & 0,3758942 & 3,793347 & 7,001421 \\
$\mathrm{H} 2=$ & 0,2469643 & 2,492248 & 4,599967 \\
$\mathrm{NH} 3=$ & 0,2413439 & 2,435529 & 4,495281 \\
$\mathrm{CO}=$ & 0,2204848 & 2,225029 & 4,10676 \\
$\mathrm{C} 2 \mathrm{H} 6=$ & 0,1211049 & 1,222134 & 2,255705 \\
$\mathrm{C} 2 \mathrm{H} 4=$ & 0,03587345 & 0,362018 & 0,6681803 \\
$\mathrm{CO} 2=$ & 0,03200336 & 0,3229629 & 0,5960959
\end{tabular}




$\begin{array}{llll}\mathrm{CH} 2 \mathrm{O} 2= & 0,02294971 & 0,2315977 & 0,4274623 \\ \mathrm{HCN}= & 0,02088441 & 0,2107556 & 0,3889939 \\ \mathrm{CH} 3 \mathrm{OH}= & 0,0009939731 & 0,01003071 & 0,01851379 \\ \mathrm{CH} 2 \mathrm{O}= & 7,140602 \mathrm{E}-05 & 0,0007205959 & 0,001330012 \\ \mathrm{NH} 2= & 6,182649 \mathrm{E}-05 & 0,0006239238 & 0,001151583 \\ \mathrm{H}= & 6,069322 \mathrm{E}-05 & 0,0006124875 & 0,001130475 \\ \mathrm{~N} 2 \mathrm{H} 4= & 4,556461 \mathrm{E}-05 & 0,0004598166 & 0,0008486884 \\ \mathrm{CHNO}= & 1,578617 \mathrm{E}-05 & 0,0001593066 & 0,0002940338 \\ \mathrm{CNO}= & 2,897577 \mathrm{E}-07 & 2,924098 \mathrm{E}-06 & 5,397038 \mathrm{E}-06 \\ \mathrm{~N}= & 1,326933 \mathrm{E}-08 & 1,339079 \mathrm{E}-07 & 2,471552 \mathrm{E}-07 \\ \mathrm{~N} 2 \mathrm{O}= & 3,814201 \mathrm{E}-09 & 3,849112 \mathrm{E}-08 & 7,104347 \mathrm{E}-08 \\ \mathrm{C}(\mathrm{d})= & 3,889382 \mathrm{E}-10 & 3,924982 \mathrm{E}-09 & 7,24438 \mathrm{E}-09 \\ \mathrm{NO} 2= & 7,40085 \mathrm{E}-11 & 7,46859 \mathrm{E}-10 & 1,378486 \mathrm{E}-09\end{array}$

\section{3-Azidooxetane}

$C(3,000) H(5,000) N(3,000) \mathrm{O}(1,000)$

Molecular weight $\quad=99,09$

Density of explosive $\quad=1,18 \mathrm{~g} / \mathrm{cm} 3$

Oxygen balance $\quad=-121,0908 \%$

Enthalpy of formation $\quad=2276,65 \mathrm{~kJ} / \mathrm{kg}$

Internal energy of formation $=2389,21 \mathrm{~kJ} / \mathrm{kg}$

Detonation parameters (at the C-J point) :

$\begin{array}{lll}\text { Heat of detonation } & =-4520,494 \mathrm{~kJ} / \mathrm{kg} \\ \text { Detonation temperature } & =2813,306 \mathrm{~K} \\ \text { Detonation pressure } & =11,50812 \mathrm{GPa} \\ \text { Detonation velocity } & =6307,581 \mathrm{~m} / \mathrm{s} \\ \text { Particle velocity } & =1546,179 \mathrm{~m} / \mathrm{s} \\ \text { Sound velocity } & =4761,402 \mathrm{~m} / \mathrm{s} \\ \text { Density of products } & =1,563183 \mathrm{~g} / \mathrm{cm} 3 \\ \text { Volume of products } & =0,6397202 \mathrm{~cm} 3 / \mathrm{g} \\ \text { Exponent 'Gamma' } & =3,079464 \\ \text { Moles of gaseous products } & =3,323059 \mathrm{~mol} / \mathrm{mol} \text { explosive } \\ \text { Moles of condensed products } & =2,069524 \mathrm{~mol} / \mathrm{mol} \text { explosive } \\ \text { Volume of gas at STP } & =820,1259 \mathrm{dm} 3 / \mathrm{kg} \\ \text { Mean molecular mass of gas. prod. } & =22,33921 \mathrm{~g} / \mathrm{mol} \\ \text { Mean molecular mass of cond.prod. } & =12,011 \mathrm{~g} / \mathrm{mol} \\ \text { Mean molecular mass of all prod. } & =18,37553 \mathrm{~g} / \mathrm{mol} \\ \text { Entropy of products } & =7,699582 \mathrm{~kJ} / \mathrm{kg} \mathrm{K} \\ \text { Internal energy of products } & =5715,838 \mathrm{~kJ} / \mathrm{kg}, \text { i.e. } 6,744689 \mathrm{~kJ} / \mathrm{cm} 3 \\ \text { Compression energy } & =1195,345 \mathrm{~kJ} / \mathrm{kg}, \text { i.e. } 1,410507 \mathrm{~kJ} / \mathrm{cm} 3 \\ \text { Total heat energy } & =-4520,494 \mathrm{~kJ} / \mathrm{kg} \text {, i.e. }-5,334182 \mathrm{~kJ} / \mathrm{cm} 3\end{array}$


Composition of detonation products:

\begin{tabular}{|c|c|c|c|}
\hline Products & $\mathrm{mol} / \mathrm{mol}$ & $\mathrm{mol} / \mathrm{kg}$ & Mol \% \\
\hline$C(g r)=$ & 2,069524 & 20,88466 & 38,37723 \\
\hline $\mathrm{N} 2=$ & 1,361183 & 13,73642 & 25,24176 \\
\hline $\mathrm{H} 2 \mathrm{O}=$ & 0,7013227 & 7,077419 & 13,00532 \\
\hline $\mathrm{CH} 4=$ & 0,355711 & 3,589668 & 6,596301 \\
\hline $\mathrm{NH} 3=$ & 0,2586558 & 2,610233 & 4,796511 \\
\hline $\mathrm{H} 2=$ & 0,2272663 & 2,293464 & 4,214423 \\
\hline $\mathrm{CO}=$ & 0,188192 & 1,899146 & 3,489831 \\
\hline $\mathrm{C} 2 \mathrm{H} 6=$ & 0,1228524 & 1,239769 & 2,278174 \\
\hline $\mathrm{C} 2 \mathrm{H} 4=$ & 0,03316479 & 0,3346834 & 0,6150075 \\
\hline $\mathrm{CO} 2=$ & 0,02916582 & 0,2943277 & 0,5408506 \\
\hline $\mathrm{CH} 2 \mathrm{O} 2=$ & 0,02557261 & 0,2580667 & 0,4742181 \\
\hline $\mathrm{HCN}=$ & 0,01879159 & 0,1896359 & 0,348471 \\
\hline $\mathrm{CH} 3 \mathrm{OH}=$ & 0,0009443772 & 0,00953021 & 0,01751252 \\
\hline $\mathrm{NH} 2=$ & $6,08983 E-05$ & 0,0006145571 & 0,001129297 \\
\hline $\mathrm{N} 2 \mathrm{H} 4=$ & $5,743006 E-05$ & 0,0005795571 & 0,001064982 \\
\hline$H=$ & $5,457085 \mathrm{E}-05$ & 0,0005507034 & 0,001011961 \\
\hline $\mathrm{CH} 2 \mathrm{O}=$ & $5,260582 E-05$ & 0,0005308732 & 0,0009755217 \\
\hline $\mathrm{CHNO}=$ & 1,117029E-05 & 0,0001127253 & 0,0002071418 \\
\hline $\mathrm{CNO}=$ & $2,735643 \mathrm{E}-07$ & $2,760682 \mathrm{E}-06$ & $5,072974 \mathrm{E}-06$ \\
\hline$N=$ & 1,431497E-08 & 1,444599E-07 & $2,654567 \mathrm{E}-07$ \\
\hline $\mathrm{N} 2 \mathrm{O}=$ & $3,611852 \mathrm{E}-09$ & $3,644911 \mathrm{E}-08$ & $6,697814 \mathrm{E}-08$ \\
\hline$C(d)=$ & $4,788678 \mathrm{E}-10$ & $4,832509 \mathrm{E}-09$ & $8,88012 \mathrm{E}-09$ \\
\hline $\mathrm{NO} 2=$ & 8,629467E-11 & $8,708453 \mathrm{E}-10$ & 1,600247E-09 \\
\hline
\end{tabular}

\section{Hot-plate test}

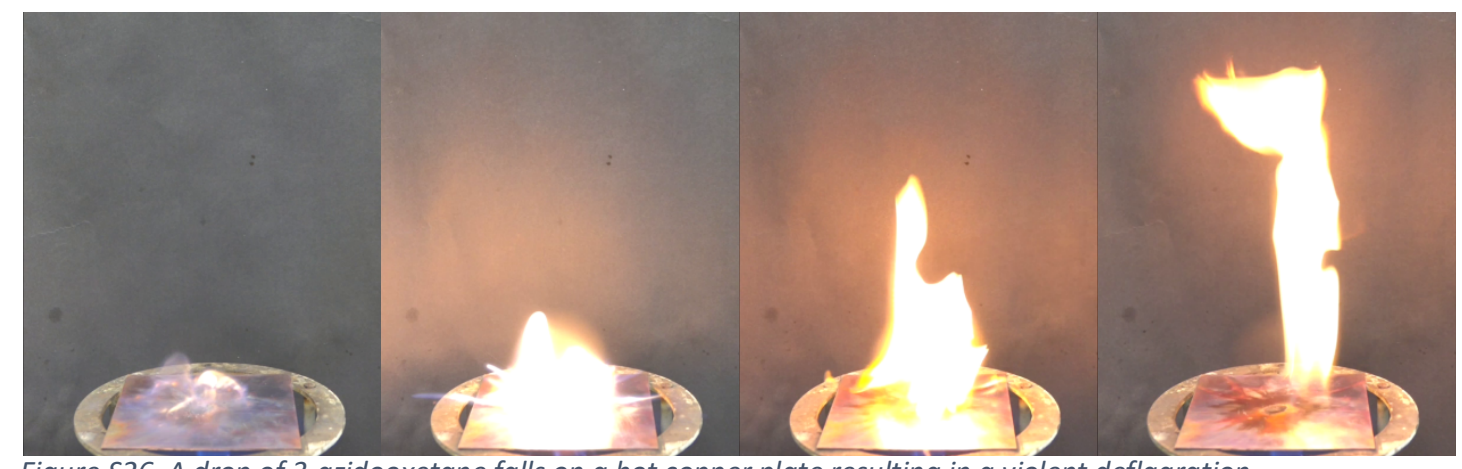

Figure S26. A drop of 3-azidooxetane falls on a hot copper plate resulting in a violent deflagration.

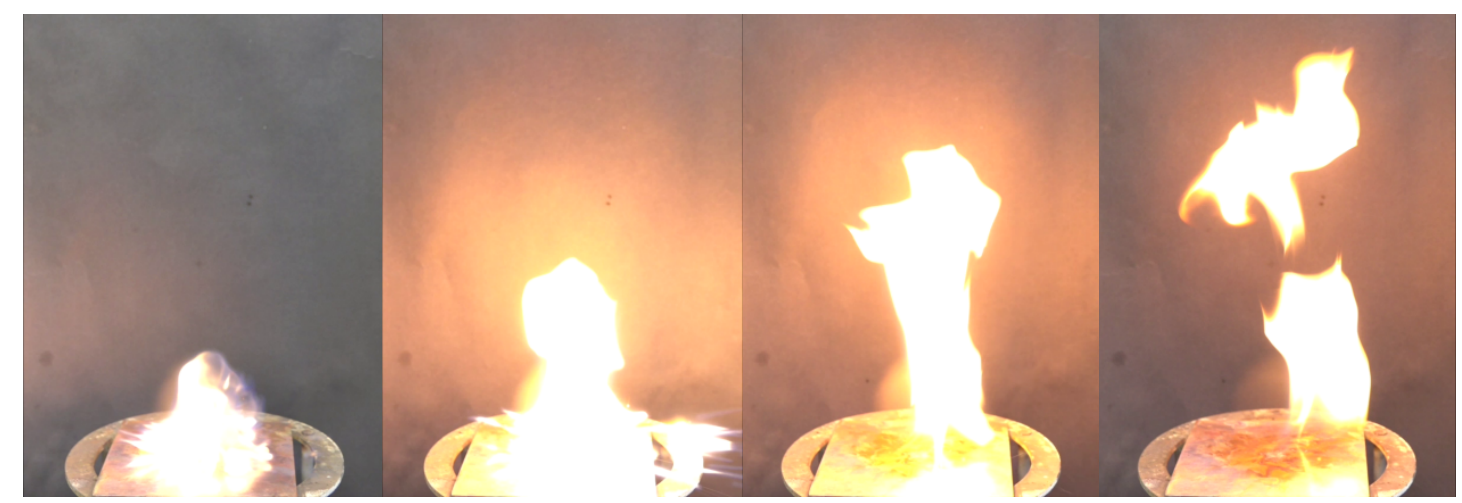

Figure S27. A drop of glycidyl azide causing a violent deflagration upon impact on a hot copper plate. 


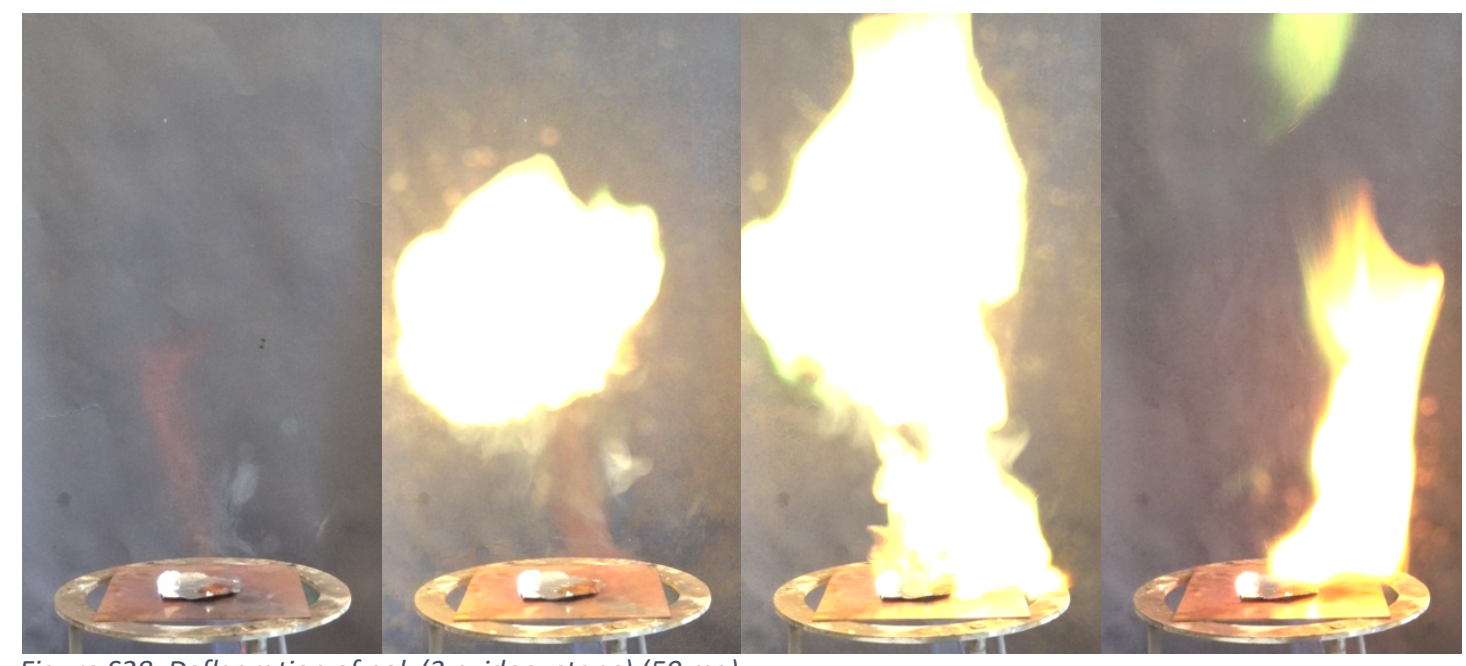

Figure S28. Deflagration of poly(3-azidooxetane) (50 mg).

\section{References}

(1) CrysAlisPRO, Oxford Diffraction /Agilent Technologies UK Ltd, Yarnton, England, 2009,

(2) Altomare, A.; Cascarano, G.; Giacovazzo, C.; Guagliardi, A.; Burla, M. C.; Polidori, G.; Camalli, M. SIR92 - a program for automatic solution of crystal structures by direct methods. J. Appl. Crystallogr. 1994, 27, 435.

(3) Altomare, A.; Burla, M. C.; Camalli, M.; Cascarano, G. L.; Giacovazzo, C.; Guagliardi, A.; Moliterni, A. G. G.; Polidori, G.; Spagna, R. SIR97: a new tool for crystal structure determination and refinement. J. Appl. Crystallogr. 1999, 32, 115.

(4) Sheldrick, G. M., Program for the Refinement of Crystal Structures, SHELXL-97, 1997.

(5) Sheldrick, G. A short history of SHELX. Acta Crystallogr., Sect. A: Found. Adv. 2008, 64, 112.

(6) Hubschle, C. B.; Sheldrick, G. M.; Dittrich, B. ShelXle: a Qt graphical user interface for SHELXL. J. Appl. Crystallogr. 2011, 44, 1281.

(7) Spek, A. Single-crystal structure validation with the program PLATON. J. Appl. Crystallogr. 2003, 36, 7.

(8) Farrugia, L. J. WinGX and ORTEP for Windows: an update. J. Appl. Crystallogr. 2012, 45, 849.

(9) M. J. Turner, J. J. McKinnon, S. K. Wolff, D. J. Grimwood, P. R. Spackman, D. Jayatilaka and M. A. Spackman, CrystalExplorer17 (2017). University of Western Australia. https://hirshfeldsurface.net. (10) Altenburg, T.; Klapötke, Thomas M.; Penger, A.; Stierstorfer, J. Two Outstanding Explosives Based on 1,2-Dinitroguanidine: Ammonium- dinitroguanidine and 1,7-Diamino-1,7-dinitrimino-2,4,6trinitro-2,4,6-triazaheptane. Z. Anorg. Allg. Chem. 2010, 636, 463.

(11) Chase, M. W., NIST-JANAF Thermochemical Tables, Fourth Edition, J. Phys. Chem. Red. Data, Monograph 9, 1998, 1.

(12) Frisch, M. J.; Trucks, G. W.; Schlegel, H. B.; Scuseria, G. E.; Robb, M. A.; Cheeseman, J. R.; Scalmani, G.; Barone, V.; Petersson, G. A.; Nakatsuji, H.; Li, X.; Caricato, M.; Marenich, A. V.; Bloino, J.; Janesko, B. G.; Gomperts, R.; Mennucci, B.; Hratchian, H. P.; Ortiz, J. V.; Izmaylov, A. F.; Sonnenberg, J. L.; Williams; Ding, F.; Lipparini, F.; Egidi, F.; Goings, J.; Peng, B.; Petrone, A.; Henderson, T.; Ranasinghe, D.; Zakrzewski, V. G.; Gao, J.; Rega, N.; Zheng, G.; Liang, W.; Hada, M.; Ehara, M.; Toyota, K.; Fukuda, R.; Hasegawa, J.; Ishida, M.; Nakajima, T.; Honda, Y.; Kitao, O.; Nakai, H.; Vreven, T.; Throssell, K.; Montgomery Jr., J. A.; Peralta, J. E.; Ogliaro, F.; Bearpark, M. J.; Heyd, J. J.; Brothers, E. N.; Kudin, K. N.; Staroverov, V. N.; Keith, T. A.; Kobayashi, R.; Normand, J.; Raghavachari, K.; Rendell, A. P.; Burant, J. C.; Iyengar, S. S.; Tomasi, J.; Cossi, M.; Millam, J. M.; Klene, M.; Adamo, C.; Cammi, R.; Ochterski, J. W.; Martin, R. L.; Morokuma, K.; Farkas, O.; Foresman, J. B.; Fox, D. J., Gaussian 16 Rev. C.01, 2016.

(13) Sućeska, M., EXPLO5, Version 6.04, 2017. 